DOI 10.4171/JEMS/391

M. Barchiesi · F. Cagnetti · N. Fusco

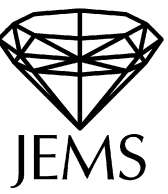

\title{
Stability of the Steiner symmetrization of convex sets
}

Received April 21, 2011 and in revised form September 8, 2011

\begin{abstract}
The isoperimetric inequality for Steiner symmetrization of any codimension is investigated and the equality cases are characterized. Moreover, a quantitative version of this inequality is proven for convex sets.
\end{abstract}

Keywords. Steiner symmetrization, quantitative isoperimetric inequality

\section{Introduction}

The present paper analyses the Steiner symmetrization of any codimension. Let $n \geq 2$ and $1 \leq k \leq n-1$. For every set $E \subset \mathbb{R}^{n}$ and every $x^{\prime} \in \mathbb{R}^{n-k}$ we define

$$
E_{x^{\prime}}:=\left\{y \in \mathbb{R}^{k}:\left(x^{\prime}, y\right) \in E\right\} \quad \text { and } L\left(x^{\prime}\right):=\mathcal{L}^{k}\left(E_{x^{\prime}}\right),
$$

where $\mathcal{L}^{k}$ stands for the outer Lebesgue measure in $\mathbb{R}^{k}$. We denote by $r\left(x^{\prime}\right)$ the radius of a $k$-dimensional ball in $\mathbb{R}^{k}$ having $\mathcal{L}^{k}$-measure equal to $L\left(x^{\prime}\right)$, and we set

$$
\pi(E)^{+}:=\left\{x^{\prime} \in \mathbb{R}^{n-k}: L\left(x^{\prime}\right)>0\right\} .
$$

Then, the Steiner symmetral of $E$ (of codimension $k$ and with respect to the subspace $y=0)$ is defined as

$$
E^{S}:=\left\{\left(x^{\prime}, y\right) \in \mathbb{R}^{n}: x^{\prime} \in \pi(E)^{+},|y| \leq r\left(x^{\prime}\right)\right\} .
$$

The importance of Steiner symmetrization has been assessed by several authors, and relies upon the fact that it acts monotonically on many geometric and analytic quantities associated with subsets of $\mathbb{R}^{n}$, e.g. the perimeter. A characterization of the sets whose perimeter is preserved under symmetrization of codimension 1 was given by Chlebík, Cianchi and Fusco [5].

M. Barchiesi, N. Fusco: Dipartimento di Matematica ed Applicazioni, Università di Napoli "Federico II", Via Cintia, 80126 Napoli, Italy; e-mail: barchies@gmail.com,n.fusco@unina.it

F. Cagnetti: Departamento de Matemática, Instituto Superior Técnico, Av. Rovisco Pais, 1049-001 Lisboa, Portugal; e-mail: cagnetti@ math.ist.utl.pt

Mathematics Subject Classification (2010): 49Q20, 52A40 
In this paper we tackle the general case $1 \leq k \leq n-1$ using a new approach, based on the regularity properties of the barycenter of the sections $E_{x^{\prime}}$ as $x^{\prime}$ varies in $\pi^{+}(E)$. The advantage of this approach is twofold. Firstly, we recover and extend the result proved in [5] for $k=1$ to any codimension, with a new and simpler proof. Secondly, we are able to obtain a quantitative isoperimetric estimate for convex sets which, to the best of our knowledge, is the first result of this kind in the framework of Steiner symmetrization.

We now provide a detailed account of the content of the paper. Our first result reads as follows.

Theorem 1.1. Let $E$ be a set of finite perimeter in $\mathbb{R}^{n}$. Then

$$
P\left(E^{S} ; B \times \mathbb{R}^{k}\right) \leq P\left(E ; B \times \mathbb{R}^{k}\right)
$$

for every Borel set $B \subset \mathbb{R}^{n-k}$. Moreover, if $P\left(E^{S}\right)=P(E)$, then either $E$ is equivalent to $\mathbb{R}^{n}$, or $\mathcal{L}^{n}(E)<\infty$ and for $\mathcal{L}^{n-k}$-a.e. $x^{\prime} \in \pi(E)^{+}$,

(a) $E_{x^{\prime}}$ is equivalent to a $k$-dimensional ball and $\mathcal{H}^{k-1}\left(\partial^{*} E_{x^{\prime}} \triangle\left(\partial^{*} E\right)_{x^{\prime}}\right)=0$;

(b) the functions $v_{x^{\prime}}^{E}\left(x^{\prime}, \cdot\right)$ and $\left|v_{y}^{E}\right|\left(x^{\prime}, \cdot\right)$ are constant $\mathcal{H}^{k-1}$-a.e. in $\partial^{*} E_{x^{\prime}}$.

Here, $P\left(E ; B \times \mathbb{R}^{k}\right)$ denotes the perimeter of $E$ in $B \times \mathbb{R}^{k}$, and $P(E)$ stands for the perimeter of $E$ in the whole $\mathbb{R}^{n}$. The reduced boundary of $E$ is indicated with $\partial^{*} E$, and its generalized inner normal with $v^{E}$ (see Section 2). Moreover, $v_{x^{\prime}}^{E}$ and $v_{y}^{E}$ denote the first $n-k$ and the last $k$ components of $v^{E}$ respectively. Finally, $\mathcal{H}^{d}$ is the Hausdorff measure of dimension $d$.

Inequality (1.1) was already proved in [4] by approximating $E^{S}$ through a sequence of simple rearrangements (polarizations). However, the proof of properties (a)-(b) when $P(E)=P\left(E^{S}\right)$ requires a direct approach. We highlight that for $k>1$ the proof is more delicate than in the case $k=1$ studied in [5]. The reason of this extra difficulty lies in the fact that the Radon measure

$$
B \subset \mathbb{R}^{n-k} \mapsto \mu(B):=\int_{\partial^{*} E \cap\left(B \times \mathbb{R}^{k}\right) \cap\left\{v_{y}^{E}=0\right\}} v_{x^{\prime}}^{E}\left(x^{\prime}, y\right) d \mathcal{H}^{n-1}\left(x^{\prime}, y\right)
$$

has a different behavior depending on whether $k=1$ or $k>1$. In fact, when $k=1$, $\mu$ is purely singular with respect to the Lebesgue measure $\mathcal{L}^{n-1}$, while, if $k>1$, it may contain a non-trivial absolutely continuous part. A somewhat surprising example by Almgren and Lieb (see Remark 3.2) shows that when $k>1$ it may even happen that $\mu$ is absolutely continuous with respect to $\mathcal{L}^{n-k}$. In other words, when $k=1$ the projection of $\left\{x \in \partial^{*} E: v_{y}^{E}(x)=0\right\}$ (the "vertical part" of the boundary) on $\mathbb{R}^{n-k}$ is a set of zero Lebesgue measure, while if $k>1$ this projection may be smeared out on a set of positive $\mathcal{L}^{n-k}$ measure.

As observed in [5], the equality $P(E)=P\left(E^{S}\right)$ does not imply that $E$ and $E^{S}$ are equivalent. In fact, if the boundary of $E^{S}$ contains vertical parts, one can easily find a set $E$ having the same perimeter of $E^{S}$ and not equivalent to (any translate of) $E^{S}$ (see Figure 1). 

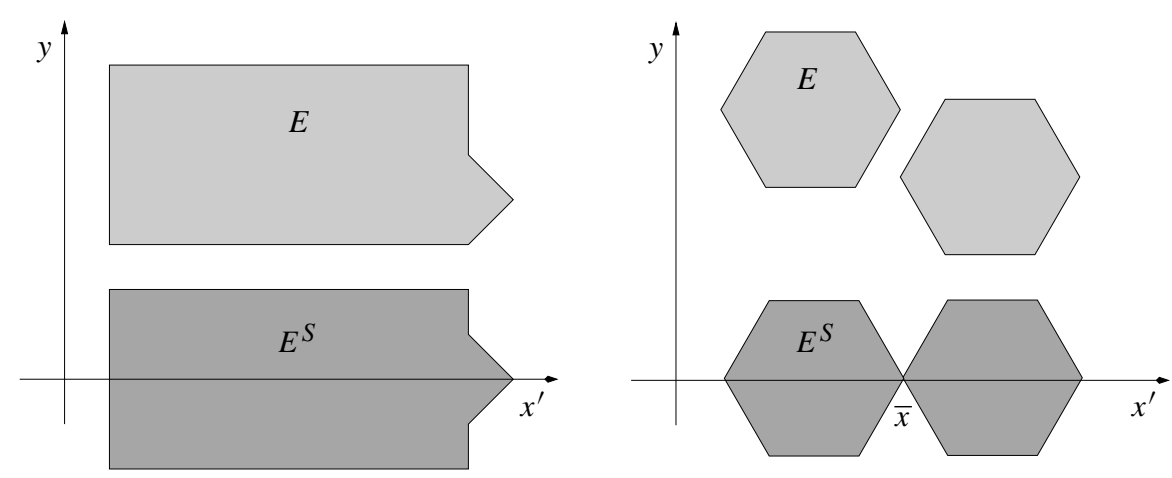

Fig. 1. Here $n=2, k=1$. On the left, the boundary of $E^{S}$ contains vertical parts, thus violating (1.2). Instead, for $E^{S}$ on the right, $L^{*}(\bar{x})=0$, violating (1.4).

Therefore, in order to characterize the equality cases, at least in a local form, we shall assume that, given an open set $\Omega \subset \mathbb{R}^{n-k}, E^{S}$ satisfies the condition

$$
\mathcal{H}^{n-1}\left(\left\{x \in \partial^{*} E^{S}: v_{y}^{E^{S}}(x)=0\right\} \cap\left(\Omega \times \mathbb{R}^{k}\right)\right)=0 .
$$

This is equivalent to the requirement $L \in W^{1,1}(\Omega)$ (see Proposition 3.5). Note that, for any set $E$ of finite perimeter, $L \in \mathrm{BV}\left(\mathbb{R}^{n-k}\right.$ ) (see Lemma 3.1). Therefore, the presence of singular parts in the measure $D L$ is equivalent to having vertical parts in the boundary of $E^{S}$. Furthermore, (1.2) is weaker than the corresponding condition on the set $E$,

$$
\mathcal{H}^{n-1}\left(\left\{x \in \partial^{*} E: v_{y}^{E}(x)=0\right\} \cap\left(\Omega \times \mathbb{R}^{k}\right)\right)=0 .
$$

However, (1.2) and (1.3) are equivalent when $P\left(E ; \Omega \times \mathbb{R}^{k}\right)=P\left(E^{S} ; \Omega \times \mathbb{R}^{k}\right)$ (see Proposition 3.6).

Condition (1.2), together with the equality of the perimeters, is not yet sufficient to conclude that $E$ and $E^{S}$ are equivalent. As shown even through simple examples (see Figure 1), this is due to the fact that the set $E^{S}$ may not be connected in a proper sense. That is, the Lebesgue representative $L^{*}$ of $L$ may vanish in a set of positive $\mathcal{H}^{n-k-1}$. measure. Therefore, we are led to assume

$$
L^{*}\left(x^{\prime}\right)>0 \text { for } \mathcal{H}^{n-k-1} \text {-a.e. } x^{\prime} \in \Omega .
$$

Then the analogue of [5, Theorem 1.3] can be established for every $1 \leq k \leq n-1$.

Theorem 1.2. Let $\Omega \subset \mathbb{R}^{n-k}$ be a connected open set, and let $E$ be a set of finite perimeter such that $P\left(E^{S} ; \Omega \times \mathbb{R}^{k}\right)=P\left(E ; \Omega \times \mathbb{R}^{k}\right)$. If (1.2) and (1.4) are satisfied, then $E \cap\left(\Omega \times \mathbb{R}^{k}\right)$ is equivalent to (a translate along $\mathbb{R}^{k}$ of) $E^{S} \cap\left(\Omega \times \mathbb{R}^{k}\right)$.

Our proof of this result significantly simplifies the one given in [5] for the case of codimension 1, which was based on a delicate density argument and on the heavy use of the notion of polarization. We have devised a different approach, based on the regularity properties of the barycenter $b: \mathbb{R}^{n-k} \rightarrow \mathbb{R}^{k}$ of the $k$-dimensional sections of $E$ (see 
Definition 4.1). The role played by the barycenter can be easily understood, observing that the sets enjoying property (a) of Theorem 1.1 are completely characterized by the functions $L$ and $b$.

We start by observing that for these sets assumptions (1.3) and (1.4) imply that the barycenter $b$ is an absolutely continuous function on almost every 1-dimensional section of the set $\Omega$ (see Theorem 4.3). Note, however, that no regularity for the barycenter can be expected if (1.3) and (1.4) are not satisfied (see Example 4.2). Using the explicit expression of the derivatives of $b$ (see (4.1)) we show that, if $E$ and $E^{S}$ have the same perimeter, these derivatives are all zero, thus proving that $b$ is constant in $\Omega$. This gives that $E$ is equivalent to a translation of $E^{S}$.

The regularity of the barycenter is an essential tool also in dealing with the second issue addressed in the present paper, namely a quantitative version of the inequality $P\left(E^{S}\right) \leq P(E)$. In recent years quantitative isoperimetric and related functional inequalities have attracted the interest of several authors $([8,11,12,13,14,15]$, to name but a few). Let us just recall here the result proved in [13]. To this end, given a set of finite perimeter $E$, we define the asymmetry index $A(E)$ and the isoperimetric deficit $\delta(E)$ of $E$ as

$$
A(E):=\min \left\{\frac{\mathcal{L}^{n}\left(E \triangle B_{r}(x)\right)}{\mathcal{L}^{n}(E)}: x \in \mathbb{R}^{n}\right\}, \quad \delta(E):=\frac{P(E)-P\left(B_{r}\right)}{P\left(B_{r}\right)},
$$

where $B_{r}(x)$ is the ball of radius $r$ centered at $x$ with $\mathcal{L}^{n}(E)=\mathcal{L}^{n}\left(B_{r}(x)\right)$, and $B_{r}$ stands for $B_{r}(0)$.

Theorem 1.3. There exists a constant $\gamma_{n}>0$ such that for every set $E \subset \mathbb{R}^{n}$ of finite perimeter,

$$
A(E) \leq \gamma_{n} \sqrt{\delta(E)}
$$

In other words, if the perimeter of $E$ is close to the one of the ball with the same volume, then there exists an optimal ball which overlaps with $E$ with the exception of a set whose measure is controlled by the square root of the difference $P(E)-P\left(B_{r}\right)$.

In this paper we prove an analogue of Theorem 1.3 for Steiner symmetrization. To state our result precisely, let us recall that the eccentricity of a bounded convex set $C$ is defined as the ratio between the outer and inner radii of $C$, i.e., the radius of the smallest ball containing $C$ and of the largest ball contained in $C$, respectively.

Let us also introduce the asymmetry index and the isoperimetric deficit of $E$ relative to its Steiner symmetral $E^{S}$ :

$$
\lambda(E):=\inf \left\{\frac{\mathcal{L}^{n}\left(E^{S} \triangle(E+(0, y))\right)}{\mathcal{L}^{n}\left(E^{S}\right)}: y \in \mathbb{R}^{k}\right\}, \quad D(E):=\frac{P(E)-P\left(E^{S}\right)}{P\left(E^{S}\right)} .
$$

We start with the case $k=1$.

Theorem 1.4. Let $k=1$. There exists a constant $c=c(n)$ such that, if $E^{S}$ is a Steiner symmetric bounded convex set with eccentricity $\mathcal{E}_{S}$, then

$$
\lambda(E) \leq c \mathcal{E}_{S}^{2} \sqrt{D(E)}
$$

for every set $E \subset \mathbb{R}^{n}$ of finite perimeter satisfying (1.3), whose 1-dimensional sections $E_{x^{\prime}}$ are segments, and whose Steiner symmetral is $E^{S}$. 
An interesting feature of inequality (1.6) is that it applies with the same constant to a large class of sets $E^{S}$. On the other hand, in light of the characterization of the equality cases, it is clear that we cannot have a quantitative estimate of this kind if we allow the boundary of $E^{S}$ to have even "almost" vertical parts (see Figure 2). Similarly, it is also clear that Theorem 1.4 cannot hold, should the vertical sections shrink in an arbitrary way (see Figure 3). Therefore, the convexity assumption on $E^{S}$ seems a natural geometric compromise to avoid both these phenomena, even though Theorem 1.4 may be proven under weaker assumptions (see Remark 5.7). Anyway, the set $E$ is not required to be convex. Note also that inequality (1.6) cannot hold with a constant not depending on the eccentricity (see Example 5.5). Finally, considering that the exponent $1 / 2$ in (1.5) is optimal, it is hardly surprising that also in our inequality we cannot replace the exponent on the right-hand side with any number larger than 1/2 (see Example 5.6).

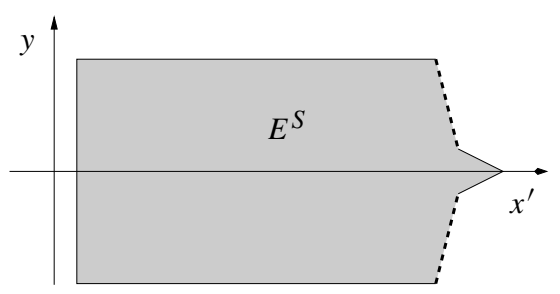

Fig. 2. When $E^{S}$ is as in the figure, the quantitative inequality $\lambda(E) \leq c \sqrt{D(E)}$ holds with a constant $c$ which goes to infinity as the slope of the two dotted segments becomes larger and larger.

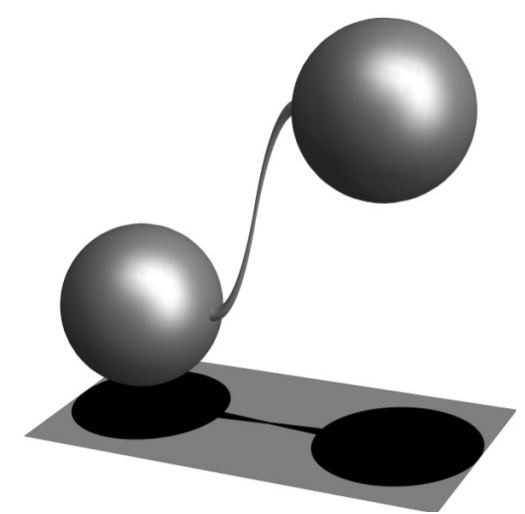

Fig. 3. Here $n=3, k=1$, and $x^{\prime}$ varies in the horizontal plane. Let $E_{\varepsilon}^{S}$ be given by the union of two balls, connected by a narrow neck whose surface measures $\varepsilon$. By lifting one of the two balls, we obtain a set $E_{\varepsilon}$ as in the figure, such that $\lambda\left(E_{\varepsilon}\right) \approx 1 / 2$, while $D\left(E_{\varepsilon}\right) \rightarrow 0$ as $\varepsilon \rightarrow 0$. Therefore, for every $\varepsilon>0$ a quantitative isoperimetric inequality $\lambda(E) \leq c_{\varepsilon} \sqrt{D(E)}$ can only hold with a constant $c_{\varepsilon} \rightarrow \infty$.

In higher codimension our result is slightly weaker, since we have to assume that the set $E$ is convex. 
Theorem 1.5. Let $2 \leq k \leq n-1$. There exists a constant $c=c(n, k)$ such that, if $E^{S}$ is a Steiner symmetric bounded convex set with eccentricity $\mathcal{E}_{S}$, then

$$
\lambda(E) \leq c \mathcal{E}_{S}^{2 n} \sqrt{D(E)}
$$

for every convex set $E \subset \mathbb{R}^{n}$ whose Steiner symmetral is $E^{S}$.

By way of conclusion, a short overview of the proof of Theorems 1.4 and 1.5 is provided. Concerning the case $k=1$, note that the convexity assumption on $E^{S}$ implies that one can estimate $\left|\nu_{y}^{E^{S}}\left(x^{\prime}\right)\right|$ by $\operatorname{dist}\left(x^{\prime}, \partial \Omega\right)$. Using this estimate, the key point of the proof is to derive the inequality

$$
\int_{\Omega}\left|v_{y}^{E^{S}}\left(x^{\prime}\right)\right|\left|\nabla b\left(x^{\prime}\right)\right| d x^{\prime} \leq c \sqrt{D(E)}
$$

from which (1.6) follows, thanks to a weighted Poincaré inequality (see Corollary 5.2) applied to the barycenter $b$. The case $k \geq 2$ is then obtained via a symmetrization argument, by applying estimate (1.6) $k$ times.

\section{Preliminary results}

In this section we recall some classical results of geometric measure theory, which will be instrumental in the forthcoming arguments. We refer the reader to the monograph [1] for a comprehensive illustration of the subject.

We first give some notation. Let $n \geq 2$ and $1 \leq k \leq n-1$. For $x \in \mathbb{R}^{n}$, we write $x=\left(x^{\prime}, y\right)$, with $x^{\prime} \in \mathbb{R}^{n-k}$ and $y \in \mathbb{R}^{k}$. Similarly, when $v=\left(v_{1}, \ldots, v_{n}\right)$ is a vector in $\mathbb{R}^{n}$, we set

$$
v_{x^{\prime}}=\left(v_{1}, \ldots, v_{n-k}\right) \in \mathbb{R}^{n-k}, \quad v_{y}=\left(v_{n-k+1}, \ldots, v_{n}\right) \in \mathbb{R}^{k} .
$$

If $D u$ is the distributional gradient of a function $u \in L_{\text {loc }}^{1}$, we write $D_{x^{\prime}} u=$ $\left(D_{1} u, \ldots, D_{n-k} u\right)$ and $D_{y} u=\left(D_{n-k+1} u, \ldots, D_{n} u\right)$. We shall indicate by $B_{r}(x)$ the open ball in $\mathbb{R}^{n}$ of radius $r$ centered at $x$. Occasionally, when balls of different dimensions come into play, we shall specify the dimension $d$ of a ball by writing $B_{r}^{d}(x)$. Finally, we simply write $B_{r}^{d}$ if the center of the ball is the origin. The measure of the unit ball in $\mathbb{R}^{d}$ will be denoted by $\omega_{d}$.

Let $\Omega$ be an open set in $\mathbb{R}^{n}$. We recall that a function $u \in L^{1}(\Omega)$ is said to be of bounded variation if its distributional derivative $D u$ is a vector-valued Radon measure in $\Omega$ with finite total variation. The set of all functions of bounded variation in $\Omega$ will be denoted by $\mathrm{BV}(\Omega)$. The space $\mathrm{BV}_{\text {loc }}(\Omega)$ of functions of locally bounded variation is defined accordingly. If $u \in \mathrm{BV}(\Omega)$, its distributional gradient can be split into the sum of an absolutely continuous part $\nabla u \mathcal{L}^{n}$ and a singular part $D^{s} u$, with respect to the Lebesgue measure. That is,

$$
D u=\nabla u \mathcal{L}^{n}+D^{s} u .
$$

If $u \in \mathrm{BV}(\Omega)$ and $D^{s} u=0$, then $u$ belongs to the Sobolev space $W^{1,1}(\Omega)$. We recall that in this case the Lebesgue representative $u^{*}$ exists for $\mathcal{H}^{n-1}$-a.e. point in $\Omega$. 
If $E$ is a Borel set, we say that it has locally finite perimeter in $\Omega$ if its characteristic function $\chi_{E}$ belongs to $\mathrm{BV}_{\text {loc }}(\Omega)$. If the total variation $\left|D \chi_{E}\right|(\Omega)$ is finite, $E$ is said to be a set of finite perimeter in $\Omega$, or simply a set of finite perimeter if $\Omega=\mathbb{R}^{n}$. For a set of finite perimeter, we define the reduced boundary $\partial^{*} E$ of $E$ as the set of all points $x \in \mathbb{R}^{n}$ such that

$$
v^{E}(x):=\lim _{r \rightarrow 0^{+}} \frac{D \chi_{E}\left(B_{r}(x)\right)}{\left|D \chi_{E}\right|\left(B_{r}(x)\right)} \quad \text { exists and belongs to } \mathbb{S}^{n-1} .
$$

We shall refer to the vector $v^{E}(x)$ as the (generalized) inner normal to $\partial^{*} E$ at $x$. From the Besicovitch derivation theorem and [1, Theorem 3.59], it follows that $\partial^{*} E$ is $(n-1)$ rectifiable and

$$
D \chi_{E}=v^{E} \mathcal{H}^{n-1}\left\lfloor\partial^{*} E .\right.
$$

Given any Borel set $B \subset \mathbb{R}^{n}$, the perimeter of $E$ in $B$ is defined as

$$
P(E ; B):=\left|D \chi_{E}\right|(B)=\mathcal{H}^{n-1}\left(\partial^{*} E \cap B\right),
$$

where the second equality follows from (2.1). When $B=\mathbb{R}^{n}$, we shall simply write $P(E)$, the perimeter of $E$. In the following, given a measurable set $E$, and $0 \leq a \leq 1$,

$$
E^{(a)}:=\left\{x \in \mathbb{R}^{n}: \lim _{r \rightarrow 0^{+}} \frac{\mathcal{L}^{n}\left(E \cap B_{r}(x)\right)}{\omega_{n} r^{n}}=a\right\}
$$

will denote the set of points with density a with respect to $E$. The next result establishes the connection between reduced boundary and densities (see [1, Theorem 3.61]).

Theorem 2.1. Let $E \subset \mathbb{R}^{n}$ be a set of finite perimeter. Then

$\partial^{*} E \subset E^{(1 / 2)}, \quad \mathcal{H}^{n-1}\left(E^{(1 / 2)} \backslash \partial^{*} E\right)=0, \quad \mathcal{H}^{n-1}\left(\mathbb{R}^{n} \backslash\left(E^{(0)} \cup E^{(1)} \cup E^{(1 / 2)}\right)\right)=0$.

The next proposition is a special case of the coarea formula (see [1, Theorem 2.93]).

Proposition 2.2. Let $E$ be a set of finite perimeter in $\mathbb{R}^{n}$ and let $g: \mathbb{R}^{n} \rightarrow[0, \infty]$ be a Borel function. Then

$$
\int_{\partial^{*} E} g(x)\left|v_{y}^{E}(x)\right| d \mathcal{H}^{n-1}(x)=\int_{\mathbb{R}^{n-k}} d x^{\prime} \int_{\left(\partial^{*} E\right)_{x^{\prime}}} g\left(x^{\prime}, y\right) d \mathcal{H}^{k-1}(y) .
$$

The following proposition gives a link between the $k$-dimensional sections $y \mapsto u_{x^{\prime}}(y)$ and the total variation of the vector measure $D_{y} u$. Its proof can be obtained as in [1, Theorem 3.103].

Proposition 2.3. Let $\Omega \subset \mathbb{R}^{n}$ be open, and let $u \in L_{\text {loc }}^{1}(\Omega)$. For every $x^{\prime} \in \mathbb{R}^{n-k}$ set $u_{x^{\prime}}(\cdot):=u\left(x^{\prime}, \cdot\right)$. Then

$$
\left|D_{y} u\right|(\Omega)=\int_{\mathbb{R}^{n-k}}\left|D u_{x^{\prime}}\right|\left(\Omega_{x^{\prime}}\right) d x^{\prime} .
$$

Finally, we prove an enhanced version of a result by Vol'pert [17]. We consider this result to be of some interest in itself, regardless of its applications. 
Theorem 2.4 (Vol'pert). Let $E$ be a set of finite perimeter in $\mathbb{R}^{n}$. Then, for $\mathcal{L}^{n-k}$-a.e. $x^{\prime} \in \mathbb{R}^{n-k}$,

(i) $E_{x^{\prime}}$ is a set of finite perimeter in $\mathbb{R}^{k}$;

(ii) $\mathcal{H}^{k-1}\left(\partial^{*}\left(E_{x^{\prime}}\right) \triangle\left(\partial^{*} E\right)_{x^{\prime}}\right)=0$;

(iii) For $\mathcal{H}^{k-1}$-a.e. s such that $\left(x^{\prime}, s\right) \in\left(\partial^{*} E\right)_{x^{\prime}} \cap \partial^{*}\left(E_{x^{\prime}}\right)$ :

(a) $v_{y}^{E}\left(x^{\prime}, s\right) \neq 0$;

(b) $v_{y}^{E}\left(x^{\prime}, s\right)=v^{E_{x^{\prime}}}(s)\left|v_{y}^{E}\left(x^{\prime}, s\right)\right|$

In particular, there exists a Borel set $G_{E} \subset \pi(E)^{+}$such that $\mathcal{L}^{n-k}\left(\pi(E)^{+} \backslash G_{E}\right)=0$ and (i)-(iii) are satisfied for every $x^{\prime} \in G_{E}$.

Proof. By Proposition 2.3, for every open set $\Omega \subset \mathbb{R}^{n}$ we have

$$
\int_{\mathbb{R}^{n-k}}\left|D \chi_{E_{x^{\prime}}}\right|\left(\Omega_{x^{\prime}}\right) d x^{\prime}=\left|D_{y} \chi_{E}\right|(\Omega) .
$$

Since $E$ has finite perimeter, this relation with $\Omega=\mathbb{R}^{n}$ gives $\int_{\mathbb{R}^{n-k}}\left|D \chi_{E_{x^{\prime}}}\right|\left(\mathbb{R}^{k}\right) d x^{\prime}<\infty$ and of course this implies (i).

To prove (ii), let us set

$$
\mathcal{M}:=\left\{B \subset \mathbb{R}^{n}: B \text { is a Borel set such that (2.3) holds with } \Omega \text { replaced by } B\right\} .
$$

One can check that

(a) $\left\{B_{h}\right\}_{h \in \mathbb{N}} \subset \mathcal{M}, B_{h} \nearrow B \Rightarrow B \in \mathcal{M}$;

(b) $B, B^{\prime}, B \cup B^{\prime} \in \mathcal{M} \Rightarrow B \cap B^{\prime} \in \mathcal{M}$;

(c) $B \in \mathcal{M} \Rightarrow \mathbb{R}^{n} \backslash B \in \mathcal{M}$.

Since $\mathcal{M}$ contains all the open subsets of $\mathbb{R}^{n}$, from [1, Remark 1.9] we know that $\mathcal{M}$ coincides with the Borel $\sigma$-algebra in $\mathbb{R}^{n}$, that is, (2.3) holds true for all Borel sets. Thus,

$$
\begin{aligned}
& \int_{\partial^{*} E} \chi_{B}(x)\left|v_{y}^{E}(x)\right| d \mathcal{H}^{n-1}(x)=\left|D_{y} \chi_{E}\right|(B)=\int_{\mathbb{R}^{n-k}}\left|D \chi_{E_{x^{\prime}}}\right|\left(B_{x^{\prime}}\right) d x^{\prime} \\
& =\int_{\mathbb{R}^{n-k}} \mathcal{H}^{k-1}\left(\partial^{*}\left(E_{x^{\prime}}\right) \cap B_{x^{\prime}}\right) d x^{\prime}=\int_{\mathbb{R}^{n-k}} d x^{\prime} \int_{\partial^{*}\left(E_{x^{\prime}}\right)} \chi_{B}\left(x^{\prime}, y\right) d \mathcal{H}^{k-1}(y) .
\end{aligned}
$$

On the other hand, by Proposition 2.2,

$$
\int_{\partial^{*} E} \chi_{B}(x)\left|v_{y}^{E}(x)\right| d \mathcal{H}^{n-1}(x)=\int_{\mathbb{R}^{n-k}} d x^{\prime} \int_{\left(\partial^{*} E\right)_{x^{\prime}}} \chi_{B}\left(x^{\prime}, y\right) d \mathcal{H}^{k-1}(y) .
$$

Let $\left\{C^{h}\right\}_{h \in \mathbb{N}}$ be a countable base for the Borel $\sigma$-algebra in $\mathbb{R}^{k}$ and $A$ any Borel set in $\mathbb{R}^{n-k}$. By comparing (2.4) and (2.5) with $B=A \times C^{h}$, for every $h \in \mathbb{N}$ and for $\mathcal{L}^{n-k}$-a.e. $x^{\prime} \in \mathbb{R}^{n-k}$ we get

$$
\int_{\partial^{*}\left(E_{x^{\prime}}\right)} \chi_{C^{h}}(y) d \mathcal{H}^{k-1}(y)=\int_{\left(\partial^{*} E\right)_{x^{\prime}}} \chi_{C^{h}}(y) d \mathcal{H}^{k-1}(y),
$$

and then the arbitrariness of $C^{h}$ immediately implies (ii). 
By applying (2.4) to the Borel set $Z:=\left\{x \in \partial^{*} E: v_{y}^{E}(x)=0\right\}$, taking into account (ii), we get

$$
\int_{\mathbb{R}^{n-k}} d x^{\prime} \int_{\partial^{*}\left(E_{x^{\prime}}\right)} \chi Z\left(x^{\prime}, y\right) d \mathcal{H}^{k-1}(y)=\int_{\mathbb{R}^{n-k}} d x^{\prime} \int_{\left(\partial^{*} E\right)_{x^{\prime}}} \chi_{Z}\left(x^{\prime}, y\right) d \mathcal{H}^{k-1}(y)=0 .
$$

Hence, $\mathcal{H}^{k-1}\left(Z_{x^{\prime}}\right)=0$ for $\mathcal{L}^{n-k}$-a.e. $x^{\prime} \in \mathbb{R}^{n-k}$, which is (iiia).

To show (iiib), we first prove that

$$
D_{y} \chi_{E}=\mathcal{L}^{n-k} \otimes D \chi_{E_{x^{\prime}}} .
$$

Let $\phi \in C_{c}^{1}\left(\mathbb{R}^{n}\right)$. Using the Fubini Theorem and integrating by parts, we obtain

$$
\begin{aligned}
& \int_{\mathbb{R}^{n}} \phi(x) d D_{y} \chi_{E}(x)=-\int_{\mathbb{R}^{n}} \nabla_{y} \phi\left(x^{\prime}, y\right) \chi_{E}\left(x^{\prime}, y\right) d x \\
& \quad=-\int_{\mathbb{R}^{n-k}} d x^{\prime} \int_{\mathbb{R}^{k}} \chi_{E_{x^{\prime}}}(y) \nabla_{y} \phi\left(x^{\prime}, y\right) d y=\int_{\mathbb{R}^{n-k}} d x^{\prime} \int_{\mathbb{R}^{k}} \phi\left(x^{\prime}, y\right) d D \chi_{E_{x^{\prime}}}(y) .
\end{aligned}
$$

This equality leads to (2.6).

Now, by Proposition 2.2, condition (ii), and relation (2.6), for any Borel set $B \subset \mathbb{R}^{n}$,

$$
\begin{aligned}
\int_{\mathbb{R}^{n-k}} d x^{\prime} \int_{\partial^{*}\left(E_{x^{\prime}}\right)} \chi_{B}\left(x^{\prime}, y\right) \chi_{\mathbb{R}^{k} \backslash Z_{x^{\prime}}}(y) v^{E_{x^{\prime}}}(y) d \mathcal{H}^{k-1}(y) \\
=\int_{\mathbb{R}^{n-k}} D \chi_{E_{x^{\prime}}}\left((B \backslash Z)_{x^{\prime}}\right) d x^{\prime}=D_{y} \chi_{E}(B \backslash Z) \\
=\int_{\partial^{*} E} \chi_{B \backslash Z}\left(x^{\prime}, y\right) v_{y}^{E}\left(x^{\prime}, y\right) d \mathcal{H}^{n-1}\left(x^{\prime}, y\right) \\
=\int_{\mathbb{R}^{n-k}} d x^{\prime} \int_{\partial^{*}\left(E_{x^{\prime}}\right)} \chi_{B}\left(x^{\prime}, y\right) \chi_{\mathbb{R}^{k} \backslash Z_{x^{\prime}}}(y) \frac{v_{y}^{E}\left(x^{\prime}, y\right)}{\left|v_{y}^{E}\left(x^{\prime}, y\right)\right|} d \mathcal{H}^{k-1}(y) .
\end{aligned}
$$

Again by the arbitrariness of $B$, (iiib) follows.

Remark 2.5. Since $E^{S}$ is symmetric about $\mathbb{R}^{n-k}$, it is easily checked from the definition of reduced boundary that if $\left(x^{\prime}, y\right) \in \partial^{*} E^{S}$ and $R: \mathbb{R}^{k} \rightarrow \mathbb{R}^{k}$ is a rotation, then $\left(x^{\prime}, R y\right) \in \partial^{*} E^{S}$ and

$$
v_{x^{\prime}}^{E^{S}}\left(x^{\prime}, R y\right)=v_{x^{\prime}}^{E^{S}}\left(x^{\prime}, y\right), \quad v_{y}^{E^{S}}\left(x^{\prime}, R y\right)=R\left(v_{y}^{E^{S}}\left(x^{\prime}, y\right)\right) .
$$

Therefore, (i)-(iii) of Theorem 2.4 hold in a stronger form: for every $x^{\prime} \in G_{E^{S}}$,

(i) $E_{x^{\prime}}^{S}$ is a $k$-dimensional ball;

(ii) $\partial\left(E_{x^{\prime}}^{S}\right)=\left(\partial^{*} E^{S}\right)_{x^{\prime}}$;

(iii) $)_{S}$ for every $y$ such that $\left(x^{\prime}, y\right) \in \partial\left(E_{x^{\prime}}^{S}\right)$ :

(a) $v_{y}^{E^{S}}\left(x^{\prime}, y\right) \neq 0$

(b) $v_{y}^{E^{S}}\left(x^{\prime}, y\right)=v_{x^{\prime}}^{S}(y)\left|v_{y}^{E^{S}}\left(x^{\prime}, y\right)\right|$.

Moreover,

$$
\mathcal{L}^{n-k}\left(B_{0}\right)=0
$$

where $B_{0}:=\left\{x^{\prime} \in \mathbb{R}^{n-k}: \exists y \in \mathbb{R}^{k}\right.$ such that $\left(x^{\prime}, y\right) \in \partial^{*} E^{S}$ and $\left.v_{y}^{E^{S}}\left(x^{\prime}, y\right)=0\right\}$. 
In view of the above theorem, for every set $E$ of finite perimeter we will use the same notation $\partial^{*} E_{x^{\prime}}$ to indicate the sets $\left(\partial^{*} E\right)_{x^{\prime}}$ and $\partial^{*}\left(E_{x^{\prime}}\right)$ when they coincide modulo $\mathcal{H}^{k-1}$. Occasionally, we will write $p_{E}\left(x^{\prime}\right):=\mathcal{H}^{k-1}\left(\partial^{*} E_{x^{\prime}}\right)$ to denote their common measure.

The following result is a straightforward variant of [1, Lemma 2.35].

Lemma 2.6. Let $B \subset \mathbb{R}^{n}$ be a Borel set, and let $\varphi_{h}, \varphi: B \rightarrow \mathbb{R}, h \in \mathbb{N}$, be summable Borel functions such that $\left|\varphi_{h}\right| \leq|\varphi|$ for every $h$. Then

$$
\int_{B} \sup _{h} \varphi_{h} d x=\sup \left\{\sum_{h \in H} \int_{A_{h}} \varphi_{h} d x\right\},
$$

where the supremum ranges over all finite sets $H \subset \mathbb{N}$ and all finite partitions $\left\{A_{h}\right\}_{h \in H}$ of $B$ into Borel sets.

\section{Properties of the function $L$}

This section is a collection of several properties of the function $L$, which will be used to prove Theorem 1.1. We recall that for every set $E \subset \mathbb{R}^{n}, L: \mathbb{R}^{n-k} \rightarrow[0, \infty]$ is defined as

$$
L\left(x^{\prime}\right):=\mathcal{L}^{k}\left(E_{x^{\prime}}\right) \quad \text { for every } x^{\prime} \in \mathbb{R}^{n-k} .
$$

The first important property is that when $E$ is a set of finite perimeter in $\mathbb{R}^{n}$, then either $E^{S}$ is equivalent to $\mathbb{R}^{n}$, or $L$ is a function of bounded variation in $\mathbb{R}^{n-k}$. When $L \in$ $\mathrm{BV}\left(\mathbb{R}^{n-k}\right)$, the measure $D L\left\lfloor G_{E}\right.$ is absolutely continuous with respect to $\mathcal{L}^{n-k}$, and it is possible to provide the explicit expression of its corresponding density.

Lemma 3.1. Let $E$ be any set of finite perimeter in $\mathbb{R}^{n}$. Then either $L\left(x^{\prime}\right)=\infty$ for $\mathcal{L}^{n-k}$ a.e. $x^{\prime} \in \mathbb{R}^{n-k}$, or $L\left(x^{\prime}\right)<\infty$ for $\mathcal{L}^{n-k}$-a.e. $x^{\prime} \in \mathbb{R}^{n-k}$ and $\mathcal{L}^{n}(E)<\infty$. Moreover, in the latter case, $L \in \mathrm{BV}\left(\mathbb{R}^{n-k}\right)$ and

$$
|D L|(B) \leq P\left(E ; B \times \mathbb{R}^{k}\right) \quad \text { for every Borel set } B \subset \mathbb{R}^{n-k} .
$$

In addition, for any Borel set $B \subset \mathbb{R}^{n-k}$,

$$
\begin{aligned}
D L(B)= & \int_{\partial^{*} E \cap\left(B \times \mathbb{R}^{k}\right) \cap\left\{v_{y}^{E}=0\right\}} v_{x^{\prime}}^{E}\left(x^{\prime}, y\right) d \mathcal{H}^{n-1}(x) \\
& +\int_{B} d x^{\prime} \int_{\left(\partial^{*} E\right)_{x^{\prime}} \cap\left\{v_{y}^{E} \neq 0\right\}} \frac{v_{x^{\prime}}^{E}\left(x^{\prime}, y\right)}{\left|v_{y}^{E}\left(x^{\prime}, y\right)\right|} d \mathcal{H}^{k-1}(y),
\end{aligned}
$$

$D L\left\lfloor G_{E^{S}}=\nabla L \mathcal{L}^{n-k}\right.$, and for $\mathcal{L}^{n-k}$-a.e. $x^{\prime} \in G_{E^{S}}$,

$$
\nabla L\left(x^{\prime}\right)=\mathcal{H}^{k-1}\left(\partial^{*} E_{x^{\prime}}^{S}\right) \frac{v_{x^{\prime}}^{E^{S}}\left(x^{\prime}\right)}{\left|v_{y}^{E^{S}}\left(x^{\prime}\right)\right|}=k \omega_{k} r^{k-1}\left(x^{\prime}\right) \frac{v_{x^{\prime}}^{E^{S}}\left(x^{\prime}\right)}{\left|v_{y}^{E^{S}}\left(x^{\prime}\right)\right|},
$$

where we dropped the variable $y$ for functions that are constant in $\partial^{*} E_{x^{\prime}}^{S}$. 
Proof. By arguing as in [5, Lemma 3.1] we obtain the first assertion and (3.1), while by arguing as in [5, Lemma 3.2] we find that for any Borel set $B \subset \mathbb{R}^{n-k}$,

$$
D L(B)=\int_{\partial^{*} E \cap\left(B \times \mathbb{R}^{k}\right)} v_{x^{\prime}}^{E}\left(x^{\prime}, y\right) d \mathcal{H}^{n-1}(x) .
$$

Then formula (3.2) is easily obtained by splitting the integral on the right-hand side above into integrals over $\partial^{*} E \cap\left(B \times \mathbb{R}^{k}\right) \cap\left\{v_{y}^{E}=0\right\}$ and over $\partial^{*} E \cap\left(B \times \mathbb{R}^{k}\right) \cap\left\{v_{y}^{E} \neq 0\right\}$. The latter is then evaluated using the coarea formula.

Finally, as pointed out in Remark 2.5,y both constant in $\partial E_{x^{\prime}}^{S}$. Moreover, $\left|v_{y}^{E^{S}}\left(x^{\prime}\right)\right|>0$ for all $x^{\prime} \in G_{E^{S}}$. Therefore, $\partial^{*} E^{S} \cap$ $\left(G_{E^{S}} \times \mathbb{R}^{k}\right) \cap\left\{v_{y}^{E^{S}}=0\right\}=\emptyset$, and from (3.2),

$$
D L(B)=\int_{B} \mathcal{H}^{k-1}\left(\partial^{*} E_{x^{\prime}}^{S}\right) \frac{v_{x^{\prime}}^{E^{S}}\left(x^{\prime}\right)}{\left|\nu_{y}^{E^{S}}\left(x^{\prime}\right)\right|} d x^{\prime} \quad \text { for all Borel sets } B \subset G_{E^{S}},
$$

thus proving that $D L\left\lfloor G_{E^{S}}\right.$ is absolutely continuous with respect to $\mathcal{L}^{n-k}$. Since by Theorem 2.4, $L\left(x^{\prime}\right)=0$ for $\mathcal{L}^{n-k}$-a.e. $x^{\prime} \in \mathbb{R}^{n-k} \backslash G_{E^{S}}$, we have $\nabla L=0$ in $\mathbb{R}^{n-k} \backslash G_{E^{S}}$. Then, we conclude that $D L\left\lfloor G_{E^{S}}=\nabla L \mathcal{L}^{n-k}\right.$ and that formula (3.3) holds true.

Remark 3.2. As hinted in the Introduction, if $k>1$ the measure

$$
\mu(B)=\int_{\partial^{*} E \cap\left(B \times \mathbb{R}^{k}\right) \cap\left\{v_{y}^{E}=0\right\}} v_{x^{\prime}}^{E}(x) d \mathcal{H}^{n-1}(x)
$$

may contain a non-vanishing absolutely continuous part. As an example, consider the special case where $n \geq 3$ and $E=\left\{\left(x^{\prime}, y\right) \in \mathbb{R} \times \mathbb{R}^{n-1}: 0<x^{\prime}<u(y)\right\}$, with $u: \mathbb{R}^{n-1} \rightarrow[0, \infty)$ a $C^{1}$ function with compact support. Then the Steiner symmetral $E^{S}$ of codimension $n-1$ of $E$ is $E^{S}=\left\{\left(x^{\prime}, y\right) \in \mathbb{R} \times \mathbb{R}^{n-1}: 0<x^{\prime}<u^{\#}(y)\right\}$, where $u^{\#}$ is the decreasing rearrangement of $u$ (see [7, Section 2.1]). In this case, the measure $\mu$ in (3.4) reduces to

$$
\mu(B)=-\mathcal{L}^{n-1}\left(u^{-1}(B) \cap\{\nabla u=0\}\right) .
$$

A surprising example given in [2, Section 5.1] shows that, for every $0<\alpha<1$, one can always find a $C^{n-2, \alpha}$ function $u$ with compact support in the unit cube $Q$ such that the measure in (3.5) is absolutely continuous with $\mu(Q)$ arbitrarily close to 1 . Interestingly, if $u \in C^{n-2,1}$, then $\mu$ is purely singular [2, Theorem 5.2].

Observe that, if $k=1$, the measure $\mu$ in (3.4) is purely singular. In fact, by the Vol'pert Theorem, for $\mathcal{L}^{n-1}$-a.e. $x^{\prime} \in \mathbb{R}^{n-1}$, we have $v_{y}^{E}\left(x^{\prime}, y\right) \neq 0$ for all $y$ such that $\left(x^{\prime}, y\right) \in \partial^{*} E$. Therefore, the projection of $\left\{x \in \partial^{*} E: v_{y}^{E}(x)=0\right\}$ on $\mathbb{R}^{n-1}$ has zero Lebesgue measure.

The following lemma shows that if $E$ has finite perimeter, the same is true for $E^{S}$. Moreover, it provides a first estimate of $P\left(E^{S}\right)$. 
Lemma 3.3. Let $E$ be any set of finite perimeter in $\mathbb{R}^{n}$ having finite measure. Then $E^{S}$ also has finite perimeter and

$$
P\left(E^{S} ; B \times \mathbb{R}^{k}\right) \leq|D L|(B)+\left|D_{y} \chi_{E}\right|\left(B \times \mathbb{R}^{k}\right)
$$

for every Borel set $B \subset \mathbb{R}^{n-k}$.

Proof. The proof is based on the same argument of [5, Lemma 3.5]. Consider a sequence $\left\{L_{j}\right\}_{j \in \mathbb{N}}$ of non-negative functions belonging to $C_{c}^{1}\left(\mathbb{R}^{n-k}\right)$ such that $L_{j} \rightarrow L \mathcal{L}^{n-k}$-a.e. in $\mathbb{R}^{n-k}$ and $\left|D L_{j}\right|\left(\mathbb{R}^{n-k}\right) \rightarrow|D L|\left(\mathbb{R}^{n-k}\right)$. Next, denote by $E_{j}^{S}$ and $r_{j}$ the set and the function defined as $E^{S}$ and $r$, respectively, with $L_{j}$ in place of $L$. Let $\Omega \subset \mathbb{R}^{n-k}$ be an open set and let $\phi=\left(\phi_{1}, \ldots, \phi_{n}\right) \in C_{c}^{1}\left(\Omega \times \mathbb{R}^{k}, \mathbb{R}^{n}\right)$. Define $\pi(\operatorname{supp} \phi)$ as the projection of $\operatorname{supp} \phi$ on the subspace $y=0$. First of all, let us show that for every $j \in \mathbb{N}$ we have

$$
\sum_{i=1}^{n-k} \int_{\Omega \times \mathbb{R}^{k}} \chi_{\left(E_{j}^{S}\right)_{x^{\prime}}}(y) \frac{\partial \phi_{i}}{\partial x_{i}}\left(x^{\prime}, y\right) d x \leq\left|D L_{j}\right|(\pi(\operatorname{supp} \phi))
$$

whenever $\|\phi\|_{\infty} \leq 1$. Define the function $V: \Omega \rightarrow \mathbb{R}^{n-k}$ by

$$
V_{i}\left(x^{\prime}\right):=\int_{B_{r_{j}\left(x^{\prime}\right)}^{k}} \phi_{i}\left(x^{\prime}, y\right) d y, \quad i=1, \ldots, n-k,
$$

and observe that $V_{i}$ is Lipschitz continuous with compact support. In fact, for every $x^{\prime}, x^{\prime \prime} \in \Omega$,

$$
\begin{aligned}
\mid V_{i}\left(x^{\prime \prime}\right) & -V_{i}\left(x^{\prime}\right) \mid \\
= & \left|\int_{B_{r_{j}\left(x^{\prime \prime}\right)}^{k}}\left[\phi_{i}\left(x^{\prime \prime}, y\right)-\phi_{i}\left(x^{\prime}, y\right)\right] d y+\int_{B_{r_{j}\left(x^{\prime \prime}\right)}^{k}} \phi_{i}\left(x^{\prime}, y\right) d y-\int_{B_{r_{j}\left(x^{\prime}\right)}^{k}} \phi_{i}\left(x^{\prime}, y\right) d y\right| \\
\leq & c\left|x^{\prime \prime}-x^{\prime}\right|+c\left|\mathcal{L}^{k}\left(B_{r_{j}\left(x^{\prime \prime}\right)}^{k}\right)-\mathcal{L}^{k}\left(B_{r_{j}\left(x^{\prime}\right)}^{k}\right)\right| \leq c\left|x^{\prime \prime}-x^{\prime}\right|+c\left|L_{j}\left(x^{\prime \prime}\right)-L_{j}\left(x^{\prime}\right)\right| .
\end{aligned}
$$

Thus, if $x^{\prime} \in A_{j}:=\left\{x^{\prime} \in \Omega: L_{j}\left(x^{\prime}\right)>0\right\}$, by differentiating $V_{i}$ with respect to $x_{i}$ and using spherical coordinates $(\rho, \sigma)$,

$$
\begin{aligned}
\frac{\partial V_{i}}{\partial x_{i}}\left(x^{\prime}\right)= & \frac{\partial}{\partial x_{i}} \int_{0}^{r_{j}\left(x^{\prime}\right)}\left[\int_{\mathbb{S}^{k-1}} \phi_{i}\left(x^{\prime}, \rho \sigma\right) d \sigma\right] \rho^{k-1} d \rho \\
= & {\left[r_{j}\left(x^{\prime}\right)\right]^{k-1} \frac{\partial r_{j}}{\partial x_{i}}\left(x^{\prime}\right) \int_{\mathbb{S}^{k-1}} \phi_{i}\left(x^{\prime}, r_{j}\left(x^{\prime}\right) \sigma\right) d \sigma } \\
& +\int_{0}^{r_{j}\left(x^{\prime}\right)} \rho^{k-1} \frac{\partial}{\partial x_{i}}\left[\int_{\mathbb{S}^{k-1}} \phi_{i}\left(x^{\prime}, \rho \sigma\right) d \sigma\right] d \rho \\
= & \frac{\partial L_{j}}{\partial x_{i}}\left(x^{\prime}\right) \frac{1}{k \omega_{k}} \int_{\mathbb{S}^{k-1}} \phi_{i}\left(x^{\prime}, r_{j}\left(x^{\prime}\right) \sigma\right) d \sigma+\int_{B_{r_{j}\left(x^{\prime}\right)}^{k}} \frac{\partial \phi_{i}}{\partial x_{i}}\left(x^{\prime}, y\right) d y,
\end{aligned}
$$

since $r_{j}^{k}=L_{j}\left(x^{\prime}\right) / \omega_{k}$. On the other hand, if $x^{\prime} \in \Omega \backslash A_{j}$, we have $L_{j}\left(x^{\prime}\right)=V_{i}\left(x^{\prime}\right)=0$ and then $\partial L_{j} / \partial x_{i}=\partial V_{i} / \partial x_{i}=0$ a.e. in $\Omega \backslash A_{j}$, so that (3.8) still holds. By applying the 
classical divergence theorem, $\int_{\Omega} \operatorname{div} V d x^{\prime}=0$. Thus, adding (3.8) for $i=1, \ldots, n-k$, and integrating over $\Omega$, yields

$$
\begin{aligned}
\sum_{i=1}^{n-k} \int_{\Omega \times \mathbb{R}^{k}} \chi_{\left(E_{j}^{S}\right)_{x^{\prime}}} & (y) \frac{\partial \phi_{i}}{\partial x_{i}}\left(x^{\prime}, y\right) d x \\
& =-\int_{\pi(\operatorname{supp} \phi)} \sum_{i=1}^{n-k} \frac{\partial L_{j}}{\partial x_{i}}\left(x^{\prime}\right) \frac{1}{k \omega_{k}}\left[\int_{\mathbb{S}^{k-1}} \phi_{i}\left(x^{\prime}, r_{j}\left(x^{\prime}\right) \sigma\right) d \sigma\right] d x^{\prime} \\
& =-\int_{\pi(\operatorname{supp} \phi)} \frac{1}{k \omega_{k}}\left[\int_{\mathbb{S}^{k-1}} \sum_{i=1}^{n-k} \frac{\partial L_{j}}{\partial x_{i}}\left(x^{\prime}\right) \phi_{i}\left(x^{\prime}, r_{j}\left(x^{\prime}\right) \sigma\right) d \sigma\right] d x^{\prime} \\
& \leq \int_{\pi(\operatorname{supp} \phi)} \frac{\left|\nabla L_{j}\left(x^{\prime}\right)\right|}{k \omega_{k}}\left[\int_{\mathbb{S}^{k-1}}\left\|\phi\left(x^{\prime}, r_{j}\left(x^{\prime}\right) \sigma\right)\right\|_{L^{\infty}} d \sigma\right] d x^{\prime} .
\end{aligned}
$$

If $\|\phi\|_{\infty} \leq 1$, (3.7) is obtained. Hence,

$$
\begin{aligned}
\int_{\Omega \times \mathbb{R}^{k}} \chi_{E_{j}^{S}} \operatorname{div} \phi d x & =\int_{\Omega}\left[\int_{\mathbb{R}^{k}} \chi_{\left(E_{j}^{S}\right)_{x^{\prime}}} \operatorname{div}_{x^{\prime}} \phi_{x^{\prime}} d y\right] d x^{\prime}+\int_{\Omega \times \mathbb{R}^{k}} \chi_{E_{j}^{S}} \operatorname{div}_{y} \phi_{y} d x \\
& \leq\left|D L_{j}\right|(\pi(\operatorname{supp} \phi))+\int_{\Omega \times \mathbb{R}^{k}} \chi_{E_{j}^{S}} \operatorname{div}_{y} \phi_{y} d x
\end{aligned}
$$

Integrating by parts gives

$$
\begin{aligned}
\int_{\Omega \times \mathbb{R}^{k}} \chi_{E_{j}^{S}} \operatorname{div}_{y} \phi_{y} d x & =\int_{\Omega} d x^{\prime} \int_{\partial B_{r_{j}\left(x^{\prime}\right)}^{k}} \phi_{y}\left(x^{\prime}, y\right) \cdot \frac{y}{r_{j}\left(x^{\prime}\right)} d \mathcal{H}^{k-1}(y) \\
& \leq \int_{\Omega} \mathcal{H}^{k-1}\left(\partial B_{r_{j}\left(x^{\prime}\right)}^{k}\right) d x^{\prime} .
\end{aligned}
$$

Then, by the isoperimetric inequality and coarea formula,

$\underset{j}{\limsup }\left|\int_{\Omega \times \mathbb{R}^{k}} \chi_{E_{j}^{S}} \operatorname{div}_{y} \phi_{y} d x\right| \leq \int_{\Omega} \mathcal{H}^{k-1}\left(\partial B_{r\left(x^{\prime}\right)}^{k}\right) d x^{\prime} \leq \int_{\Omega} \mathcal{H}^{k-1}\left(\partial^{*} E_{x^{\prime}}\right) d x^{\prime} \leq P(E)$.

Moreover, since $\chi_{E_{j}^{S}} \rightarrow \chi_{E^{S}} \mathcal{L}^{n}$-a.e. by the definition of $E_{j}^{S}$, and $\pi(\operatorname{supp} \phi)$ is a compact subset of $\Omega$, passing to the limsup in (3.9) as $j \rightarrow \infty$ we have

$$
\int_{\Omega \times \mathbb{R}^{k}} \chi_{E^{S}} \operatorname{div} \phi d x \leq|D L|(\pi(\operatorname{supp} \phi))+P(E),
$$

thus proving that $E^{S}$ has finite perimeter. Having obtained (3.10), we can go back to (3.9) and, passing to the limit again, we conclude that

$$
\begin{aligned}
\int_{\Omega \times \mathbb{R}^{k}} \chi_{E} \operatorname{div} \phi d x & \leq|D L|(\pi(\operatorname{supp} \phi))+\int_{\Omega \times \mathbb{R}^{k}} \chi_{E^{S}} \operatorname{div}_{y} \phi_{y} d x \\
& \leq|D L|(\Omega)+\left|D_{y} \chi_{E}\right|\left(\Omega \times \mathbb{R}^{k}\right) .
\end{aligned}
$$

From this, we infer that (3.6) holds whenever $B$ is an open set, and hence when $B$ is any Borel set. 
Proof of Theorem 1.1. If $L=\infty \mathcal{L}^{n-k}$-a.e. in $\mathbb{R}^{n-k}$, then $E^{S}$ is equivalent to $\mathbb{R}^{n}$; it follows that $P\left(E^{S} ; B \times \mathbb{R}^{k}\right)=0$ for every Borel set $B \subset \mathbb{R}^{n-k}$ and (1.1) is trivially satisfied. Otherwise, by Lemma 3.1, we may assume that $L<\infty \mathcal{L}^{n-k}$-a.e. in $\mathbb{R}^{n-k}$. Let $G_{E^{S}}$ be the set associated with $E^{S}$ as in Theorem 2.4, and let $B$ be a Borel subset of $\mathbb{R}^{n-k}$. We shall prove inequality (1.1) when either $B \subset \mathbb{R}^{n-k} \backslash G_{E^{S}}$, or $B \subset G_{E^{S}}$. The general case is obtained by splitting $B$ into $B \backslash G_{E}$ and $B \cap G_{E}$.

Firstly assume that $B \subset \mathbb{R}^{n-k} \backslash G_{E} s$. Observe that, by (2.1), Proposition 2.2 and Theorem 2.4(ii),

$$
\left|D_{y} \chi_{E^{S}}\right|\left(B \times \mathbb{R}^{k}\right)=\int_{\partial^{*} E^{S} \cap\left(B \times \mathbb{R}^{k}\right)}\left|v_{y}^{E^{S}}\right| d \mathcal{H}^{n-1}(x)=\int_{B} \mathcal{H}^{k-1}\left(\partial^{*} E_{x^{\prime}}^{S}\right) d x^{\prime} .
$$

Since $\mathcal{L}^{n-k}\left(\pi(E)^{+} \cap B\right)=\mathcal{L}^{n-k}\left(G_{E^{S}} \cap B\right)=0$, the last integral equals

$$
\int_{\left(\mathbb{R}^{n-k} \backslash \pi(E)^{+}\right) \cap B} \mathcal{H}^{k-1}\left(\partial^{*} E_{x^{\prime}}^{S}\right) d x^{\prime},
$$

and vanishes. Therefore, by (3.1) and (3.6), we obtain (1.1):

$$
P\left(E^{S} ; B \times \mathbb{R}^{k}\right) \leq|D L|(B) \leq P\left(E ; B \times \mathbb{R}^{k}\right) .
$$

Suppose now that $B \subset G_{E}$. Since $\mathcal{L}^{n-k}\left(G_{E} \triangle G_{E^{S}}\right)=0$ and since $\nu_{y}^{E}\left(x^{\prime}, y\right) \neq 0$ $\mathcal{H}^{k-1}$-a.e. in $\partial^{*} E_{x^{\prime}}$ for all $x^{\prime} \in G_{E}$, we have

$$
\begin{aligned}
& P\left(E ; B \times \mathbb{R}^{k}\right)=P\left(E ;\left(B \times \mathbb{R}^{k}\right) \cap\left\{v_{y}^{E}=0\right\}\right)+P\left(E ;\left(B \times \mathbb{R}^{k}\right) \cap\left\{v_{y}^{E} \neq 0\right\}\right) \\
& \quad=P\left(E ;\left(B \times \mathbb{R}^{k}\right) \cap\left\{v_{y}^{E}=0\right\}\right)+\int_{\partial^{*} E \cap\left(B \times \mathbb{R}^{k}\right) \cap\left\{v_{y}^{E} \neq 0\right\}} d \mathcal{H}^{n-1}(x) \\
& \quad=P\left(E ;\left(B \times \mathbb{R}^{k}\right) \cap\left\{v_{y}^{E}=0\right\}\right)+\int_{B} d x^{\prime} \int_{\partial^{*} E_{x^{\prime}}} \frac{1}{\left|v_{y}^{E}\left(x^{\prime}, y\right)\right|} d \mathcal{H}^{k-1}(y) \\
& \quad=P\left(E ;\left(B \times \mathbb{R}^{k}\right) \cap\left\{v_{y}^{E}=0\right\}\right)+\int_{B} d x^{\prime} \int_{\partial^{*} E_{x^{\prime}}} \sqrt{1+\sum_{i=1}^{n-k}\left(\frac{v_{i}^{E}\left(x^{\prime}, y\right)}{\left|v_{y}^{E}\left(x^{\prime}, y\right)\right|}\right)^{2}} d \mathcal{H}^{k-1}(y),
\end{aligned}
$$

where the second equality is due to (2.2), the third to Proposition 2.2 applied to $\chi_{B \times \mathbb{R}^{k}}(x) /\left|\nu_{y}^{E}(x)\right|$, and the fourth to the fact that $v^{E}$ is a unit vector. Now, applying Jensen's inequality to the strictly convex function

$$
f(z):=\sqrt{1+|z|^{2}}, \quad z \in \mathbb{R}^{n-k},
$$

we get

$$
\begin{aligned}
P\left(E ; B \times \mathbb{R}^{k}\right) \geq & P\left(E ;\left(B \times \mathbb{R}^{k}\right) \cap\left\{v_{y}^{E}=0\right\}\right) \\
& +\int_{B} \sqrt{\left(\int_{\partial^{*} E_{x^{\prime}}} d \mathcal{H}^{k-1}\right)^{2}+\sum_{i=1}^{n-k}\left(\int_{\partial^{*} E_{x^{\prime}}} \frac{v_{i}^{E}\left(x^{\prime}, y\right)}{\left|v_{y}^{E}\left(x^{\prime}, y\right)\right|} d \mathcal{H}^{k-1}(y)\right)^{2} d x^{\prime}} \\
= & P\left(E ;\left(B \times \mathbb{R}^{k}\right) \cap\left\{v_{y}^{E}=0\right\}\right)+\int_{B} \sqrt{p_{E}^{2}\left(x^{\prime}\right)+\left|g\left(x^{\prime}\right)\right|^{2}} d x^{\prime}
\end{aligned}
$$


where we have set, for $\mathcal{L}^{n-k}$-a.e. $x^{\prime} \in B$,

$$
g\left(x^{\prime}\right):=\int_{\partial^{*} E_{x^{\prime}}} \frac{v_{x^{\prime}}^{E}\left(x^{\prime}, y\right)}{\left|v_{y}^{E}\left(x^{\prime}, y\right)\right|} d \mathcal{H}^{k-1}(y) .
$$

Note that since $B \subset G_{E}$, from Lemma 3.1 it follows that $D L\lfloor B$ is absolutely continuous. We claim that

$$
\begin{aligned}
& \int_{B} \sqrt{p_{E}^{2}\left(x^{\prime}\right)+\left|\nabla L\left(x^{\prime}\right)\right|^{2}} d x^{\prime} \\
& \leq P\left(E ;\left(B \times \mathbb{R}^{k}\right) \cap\left\{v_{y}^{E}=0\right\}\right)+\int_{B} \sqrt{p_{E}^{2}\left(x^{\prime}\right)+\left|g\left(x^{\prime}\right)\right|^{2}} d x^{\prime} .
\end{aligned}
$$

To prove this claim note that, by duality, we can write the function $f$ in (3.12) as

$$
f(z)=\sqrt{1+|z|^{2}}=\sup _{h}\left\{z \cdot w_{h}+\sqrt{1-\left|w_{h}\right|^{2}}\right\}, \quad z \in \mathbb{R}^{n-k},
$$

where $\left\{w_{h}\right\}_{h}$ is a countable dense set in $B_{1}^{n-k}$. Let now $\left\{A_{h}\right\}_{h \in H}$ be a finite partition of $B$ into Borel sets. Recalling (3.2), we have

$$
\begin{aligned}
& \sum_{h \in H} \int_{A_{h}}\left(\nabla L\left(x^{\prime}\right) \cdot w_{h}+p_{E}\left(x^{\prime}\right) \sqrt{1-\left|w_{h}\right|^{2}}\right) d x^{\prime} \\
&=\sum_{h \in H}\left(\int_{\partial^{*} E \cap\left(A_{h} \times \mathbb{R}^{k}\right) \cap\left\{v_{y}^{E}=0\right\}} w_{h} \cdot v_{x^{\prime}}^{E}\left(x^{\prime}, y\right) d \mathcal{H}^{n-1}(x)\right. \\
&\left.+\int_{A_{h}}\left(g\left(x^{\prime}\right) \cdot w_{h}+p_{E}\left(x^{\prime}\right) \sqrt{1-\left|w_{h}\right|^{2}}\right) d x^{\prime}\right) \\
& \leq \sum_{h \in H}\left(\int_{\partial^{*} E \cap\left(A_{h} \times \mathbb{R}^{k}\right) \cap\left\{v_{y}^{E}=0\right\}}\left|v_{x^{\prime}}^{E}\left(x^{\prime}, y\right)\right| d \mathcal{H}^{n-1}(x)\right. \\
&\left.+\int_{A_{h}} p_{E}\left(x^{\prime}\right)\left(\frac{g\left(x^{\prime}\right)}{p_{E}\left(x^{\prime}\right)} \cdot w_{h}+\sqrt{1-\left|w_{h}\right|^{2}}\right) d x^{\prime}\right) \\
& \leq \sum_{h \in H}\left(P\left(E ;\left(A_{h} \times \mathbb{R}^{k}\right) \cap\left\{v_{y}^{E}=0\right\}\right)+\int_{A_{h}} p_{E}\left(x^{\prime}\right)\left(\sqrt{1+\frac{\left|g\left(x^{\prime}\right)\right|^{2}}{p_{E}^{2}\left(x^{\prime}\right)}}\right) d x^{\prime}\right) \\
&= P\left(E ;\left(B \times \mathbb{R}^{k}\right) \cap\left\{v_{y}^{E}=0\right\}\right)+\int_{B} \sqrt{p_{E}^{2}\left(x^{\prime}\right)+\left|g\left(x^{\prime}\right)\right|^{2}} d x^{\prime} .
\end{aligned}
$$

From this, applying Lemma 2.6 to the functions

$$
\varphi_{h}\left(x^{\prime}\right)=p_{E}\left(x^{\prime}\right)\left(\frac{\nabla L\left(x^{\prime}\right)}{p_{E}\left(x^{\prime}\right)} \cdot w_{h}+\sqrt{1-\left|w_{h}\right|^{2}}\right)
$$


we have (3.14). Note that when $E=E^{S}$, Remark 2.5 and Lemma 3.1 lead to

$$
\begin{aligned}
P\left(E^{S} ; B \times \mathbb{R}^{k}\right) & =\int_{B} d x^{\prime} \int_{\partial^{*} E_{x^{\prime}}^{S}} \sqrt{1+\sum_{i=1}^{n-k}\left(\frac{v_{i}^{E^{S}}\left(x^{\prime}, y\right)}{\left|v_{y}^{E^{S}}\left(x^{\prime}, y\right)\right|}\right)^{2}} d \mathcal{H}^{k-1}(y) \\
& =\int_{B} d x^{\prime} \int_{\partial^{*} E_{x^{\prime}}^{S}} \sqrt{1+\frac{\left|\nabla L\left(x^{\prime}\right)\right|^{2}}{\left|\mathcal{H}^{k-1}\left(\partial B_{r\left(x^{\prime}\right)}^{k}\right)\right|^{2}}} d \mathcal{H}^{k-1}(y) \\
& =\int_{B} \sqrt{p_{E^{S}}^{2}\left(x^{\prime}\right)+\left|\nabla L\left(x^{\prime}\right)\right|^{2}} d x^{\prime}
\end{aligned}
$$

Owing to the isoperimetric inequality in $\mathbb{R}^{k}$, we have $p_{E}\left(x^{\prime}\right) \leq p_{E}\left(x^{\prime}\right)$ for $\mathcal{L}^{n-k}$-a.e. $x^{\prime} \in \mathbb{R}^{n-k}$. Hence, combining (3.13)-(3.15) yields

$$
\begin{aligned}
& P\left(E^{S} ; B \times \mathbb{R}^{k}\right) \leq \int_{B} \sqrt{p_{E}^{2}\left(x^{\prime}\right)+\left|\nabla L\left(x^{\prime}\right)\right|^{2}} d x^{\prime} \\
& \quad \leq P\left(E ;\left(B \times \mathbb{R}^{k}\right) \cap\left\{v_{y}^{E}=0\right\}\right)+\int_{B} \sqrt{p_{E}^{2}\left(x^{\prime}\right)+\left|g\left(x^{\prime}\right)\right|^{2}} d x^{\prime} \leq P\left(E ; B \times \mathbb{R}^{k}\right),
\end{aligned}
$$

proving (1.1).

Now, we move on to the case of equality. If $P(E)=P\left(E^{S}\right)$, then inequality (1.1) implies

$$
P\left(E^{S} ; B \times \mathbb{R}^{k}\right)=P\left(E ; B \times \mathbb{R}^{k}\right)
$$

for every Borel set $B \subset \mathbb{R}^{n-k}$. On the other hand, as shown above, by taking $B=G_{E^{S}}$, we have

$$
\begin{aligned}
P\left(E^{S} ; G_{E^{S}} \times \mathbb{R}^{k}\right) & =\int_{G_{E} S} \sqrt{p_{E^{S}}^{2}\left(x^{\prime}\right)+\left|\nabla L\left(x^{\prime}\right)\right|^{2}} d x^{\prime} \\
& \leq \int_{G_{E} S} \sqrt{p_{E}^{2}\left(x^{\prime}\right)+\left|\nabla L\left(x^{\prime}\right)\right|^{2}} d x^{\prime} \\
& \leq P\left(E ;\left(G_{E^{S}} \times \mathbb{R}^{k}\right) \cap\left\{v_{y}^{E}=0\right\}\right)+\int_{G_{E} S} \sqrt{p_{E}^{2}\left(x^{\prime}\right)+\left|g\left(x^{\prime}\right)\right|^{2}} d x^{\prime} \\
& \leq P\left(E ; G_{E^{S}} \times \mathbb{R}^{k}\right) .
\end{aligned}
$$

All inequalities in this chain must therefore be equalities. The first of these entails that $p_{E}\left(x^{\prime}\right)=p_{E}\left(x^{\prime}\right)$ for $\mathcal{L}^{n-k}$-a.e. $x^{\prime} \in \mathbb{R}^{n-k}$, implying that $E_{x^{\prime}}$ is equivalent to a $k$ dimensional ball of radius $r\left(x^{\prime}\right)$.

The fact that the second inequality is also an equality implies that the Jensen inequality in (3.13) is an equality too. By the strict convexity of the function in (3.12), this also implies that for $\mathcal{L}^{n-k}$-a.e. $x^{\prime} \in G_{E}$,

$$
y \mapsto \frac{v_{x^{\prime}}^{E}\left(x^{\prime}, y\right)}{\left|v_{y}^{E}\left(x^{\prime}, y\right)\right|} \text { is } \mathcal{H}^{k-1} \text {-a.e. constant in } \partial^{*} E_{x^{\prime}} .
$$


Since $v^{E}$ is a unit vector, we also see that for $\mathcal{L}^{n-k}$-a.e. $x^{\prime} \in G_{E^{S}}$,

$$
y \mapsto\left|v_{y}^{E}\left(x^{\prime}, y\right)\right| \text { is } \mathcal{H}^{k-1} \text {-a.e. constant in } \partial^{*} E_{x^{\prime}},
$$

so that (b) follows.

An inspection of the above proof leads to the following result, which, regardless of its relation to Theorem 1.1, is of some independent interest.

Proposition 3.4. Let $E \subset \mathbb{R}^{n}$ be a set of finite measure and perimeter, and let $f$ : $\mathbb{R}^{n-k} \rightarrow[0, \infty]$ be a Borel function. Then

$$
\begin{array}{rl}
\int_{\partial^{*} E} & f\left(x^{\prime}\right) d \mathcal{H}^{n-1}(x) \\
& \geq \int_{\mathbb{R}^{n-k}} f\left(x^{\prime}\right) \sqrt{p_{E}^{2}\left(x^{\prime}\right)+\left|\nabla L\left(x^{\prime}\right)\right|^{2}} d x^{\prime}+\int_{\mathbb{R}^{n-k}} f\left(x^{\prime}\right) d\left|D^{s} L\right|\left(x^{\prime}\right),
\end{array}
$$

with equality if $E=E^{S}$.

Proof. It is enough to show (3.16) for $f=\chi_{B}$ with $B \subset \mathbb{R}^{n-k}$ an arbitrary Borel set.

In the case $B \subset \mathbb{R}^{n-k} \backslash G_{E^{S}}$, thanks to Lemma 3.1,

$$
|D L|(B)=\left|D^{s} L\right|(B) .
$$

Therefore, (3.16) follows from (3.1), on observing that $p_{E}$ vanishes $\mathcal{L}^{n-k}$-a.e. in $B$. Conversely, when $B \subset G_{E}$, by Lemma 3.1 we have $\left|D^{s} L\right|(B)=0$, and (3.16) follows from (3.13) and (3.14).

In the case $E=E^{S}$, the opposite of inequality (3.16) follows from (3.15) when $B \subset G_{E^{S}}$, and from (3.11) and (3.17) when $B \subset \mathbb{R}^{n-k} \backslash G_{E^{S}}$.

We conclude this section by providing two additional results related to conditions (1.2) and (1.3), which extend [5, Propositions 1.2 and 4.2], respectively. The proof of Proposition 3.5 below uses arguments similar to the ones in [5]. However, the proof of Proposition 3.6 requires a different idea, due to the phenomena occurring when $k>1$ and described in the Introduction.

Proposition 3.5. Let $\Omega$ be an open subset of $\mathbb{R}^{n-k}$ and let $E$ be any set of finite perimeter in $\mathbb{R}^{n}$, with $\mathcal{L}^{n}\left(E \cap\left(\Omega \times \mathbb{R}^{k}\right)\right)<\infty$. Then the following conditions are equivalent:

(i) $\mathcal{H}^{n-1}\left(\left\{x \in \partial^{*} E^{S}: v_{y}^{E S}(x)=0\right\} \cap\left(\Omega \times \mathbb{R}^{k}\right)\right)=0$;

(ii) $L \in W^{1,1}(\Omega)$;

(iii) $P\left(E^{S} ; B \times \mathbb{R}^{k}\right)=0$ for every Borel set $B \subset \Omega$ such that $\mathcal{L}^{n-k}(B)=0$.

Proof. (i) $\Rightarrow$ (ii). This follows by observing that, by (3.2), $D L(B)=0$ if $B \subset \mathbb{R}^{n-k}$ is a Borel set with zero $\mathcal{L}^{n-k}$-measure.

(ii) $\Rightarrow$ (iii). This is a consequence of the fact that for $E^{S}$ equality holds in (3.16).

(iii) $\Rightarrow$ (i). From (2.7) it follows that

$$
\mathcal{H}^{n-1}\left(\left\{x \in \partial^{*} E^{S}: v_{y}^{E^{S}}(x)=0\right\} \cap\left(\Omega \times \mathbb{R}^{k}\right)\right) \leq P\left(E^{S} ; B_{0} \times \mathbb{R}^{k}\right)=0 .
$$


Proposition 3.6. Let $E$ and $\Omega$ be as in Proposition 3.5. If

$$
\mathcal{H}^{n-1}\left(\left\{x \in \partial^{*} E: v_{y}^{E}(x)=0\right\} \cap\left(\Omega \times \mathbb{R}^{k}\right)\right)=0,
$$

then

$$
\mathcal{H}^{n-1}\left(\left\{x \in \partial^{*} E^{S}: v_{y}^{E^{S}}(x)=0\right\} \cap\left(\Omega \times \mathbb{R}^{k}\right)\right)=0 .
$$

Conversely, if E satisfies $P\left(E ; \Omega \times \mathbb{R}^{k}\right)=P\left(E^{S} ; \Omega \times \mathbb{R}^{k}\right)$ and (3.19) holds, then so does (3.18).

Proof. If (3.18) holds, then arguing as in the proof of (i) $\Rightarrow$ (ii) in Proposition 3.5, we find that $L \in W^{1,1}(\Omega)$. Therefore, (3.19) follows by Proposition 3.5 again.

Let us now show that (3.19) implies (3.18) when $P\left(E ; \Omega \times \mathbb{R}^{k}\right)=P\left(E^{S} ; \Omega \times \mathbb{R}^{k}\right)$. First, we recall that this implication is proven in [5, Lemma 4.2] when $k=1$. So, we have only to deal with the case $k>1$.

We start by proving that if $F \subset \mathbb{R}^{n}$ is a set of finite perimeter satisfying (1.3) and such that almost every section $F_{X^{\prime}}$ is a $k$-dimensional ball, then

$$
\mathcal{H}^{n-1}\left(\left\{x \in \partial^{*} F: v_{y_{i}}^{F}(x)=0\right\} \cap\left(\Omega \times \mathbb{R}^{k}\right)\right)=0, \quad i=1, \ldots, k .
$$

Set $B^{i}:=\left\{x \in \partial^{*} F: v_{y_{i}}^{F}(x)=0\right\} \cap\left(\Omega \times \mathbb{R}^{k}\right)$. From Theorem 2.4, $B_{x^{\prime}}^{i}=\left\{y \in \partial^{*} F_{x^{\prime}}\right.$ : $\left.v_{y_{i}} F_{x^{\prime}}(y)=0\right\}$ up to an $\mathcal{H}^{k-1}$-negligible set. Since $F_{x^{\prime}}$ is (equivalent to) a $k$-dimensional ball, $\mathcal{H}^{k-1}\left(B_{x^{\prime}}^{i}\right)=0$. From (1.3) and using the coarea formula, we have

$\mathcal{H}^{n-1}\left(B^{i}\right)=\mathcal{H}^{n-1}\left(B^{i} \cap\left\{x \in \partial^{*} F: v_{y}^{F}(x) \neq 0\right\}\right)=\int_{\mathbb{R}^{k}} d x^{\prime} \int_{\partial^{*} F_{x^{\prime}} \cap B_{x^{\prime}}^{i}} \frac{d \mathcal{H}_{y}^{k-1}(y)}{\left.\mid x^{\prime}, y\right) \mid}=0$.

Set $E^{S_{0}}=E$ and for every $i=1, \ldots, k$, denote by $E^{S_{i}}$ the (1-codimensional) Steiner symmetral of $E^{S_{i-1}}$ with respect to the hyperplane $y_{i}=0$. Observe now that, by Theorem 1.1, almost every section $E_{x^{\prime}}$ of $E$ is a $k$-dimensional ball. Then, clearly, $E^{S_{k}}$ is equivalent to $E^{S}$ in $\Omega \times \mathbb{R}^{k}$. By applying repeatedly Theorem 1.1 (for the 1-codimensional Steiner symmetrization) we get

$$
P\left(E^{S} ; \Omega \times \mathbb{R}^{k}\right)=P\left(E^{S_{k-1}} ; \Omega \times \mathbb{R}^{k}\right)=\cdots=P\left(E^{S_{1}} ; \Omega \times \mathbb{R}^{k}\right)=P\left(E ; \Omega \times \mathbb{R}^{k}\right) .
$$

From assumption (3.19) and (3.20), $\mathcal{H}^{n-1}\left(\left\{x \in \partial^{*} E^{S}: v_{y_{k}}^{E^{S}}(x)=0\right\} \cap\left(\Omega \times \mathbb{R}^{k}\right)\right)=0$. Therefore, since the assertion is true for $k=1$, we deduce that $\mathcal{H}^{n-1}\left(\left\{x \in \partial^{*} E^{S_{k-1}}\right.\right.$ : $\left.\left.v_{y_{k}}^{E^{S_{k-1}}}(x)=0\right\} \cap\left(\Omega \times \mathbb{R}^{k}\right)\right)=0$ and, a fortiori, $\mathcal{H}^{n-1}\left(\left\{x \in \partial^{*} E^{S_{k-1}}: v_{y}^{E^{S_{k-1}}}(x)=0\right\}\right.$ $\left.\cap\left(\Omega \times \mathbb{R}^{k}\right)\right)=0$. Iterating this argument yields (3.18). 


\section{Regularity of the barycenter of the sections}

The next definition has an important role in describing the properties of a set of finite perimeter.

Definition 4.1. The barycenter of the sections of a set $E \subset \mathbb{R}^{n}$ is the function $b$ : $\mathbb{R}^{n-k} \rightarrow \mathbb{R}^{k}$ defined as

$$
b\left(x^{\prime}\right):= \begin{cases}\frac{1}{L\left(x^{\prime}\right)} \int_{E_{x^{\prime}}} y d y & \text { if } 0<L\left(x^{\prime}\right)<\infty \text { and }|y| \in L^{1}\left(E_{x^{\prime}}\right), \\ 0 & \text { otherwise. }\end{cases}
$$

The following example shows that in general $b$ is not summable, even if $E$ is a set of finite perimeter.

Example 4.2. Let $n=3, k=2$. Set

$$
E=\left\{\left(x^{\prime}, y\right) \in \mathbb{R} \times \mathbb{R}^{2}:\left|y-b\left(x^{\prime}\right)\right|<r\left(x^{\prime}\right), x^{\prime} \in(-1,1)\right\},
$$

where $b\left(x^{\prime}\right)=\left(0,1 /\left|x^{\prime}\right|\right)$ and $r\left(x^{\prime}\right)=\left|x^{\prime}\right|^{2}$. Then

$$
\begin{aligned}
P\left(E ;(-1,1) \times \mathbb{R}^{2}\right) & =2 \int_{0}^{1} r\left(x^{\prime}\right) d x^{\prime} \int_{0}^{2 \pi} \sqrt{1+\left|b^{\prime}\left(x^{\prime}\right) \cdot(\cos \theta, \sin \theta)+r^{\prime}\left(x^{\prime}\right)\right|^{2}} d \theta \\
& =2 \int_{0}^{1} r\left(x^{\prime}\right) d x^{\prime} \int_{0}^{2 \pi} \sqrt{1+\left|-\frac{1}{\left|x^{\prime}\right|^{2}} \sin \theta+2 x^{\prime}\right|^{2}} d \theta<\infty .
\end{aligned}
$$

Therefore, $E$ is a set of finite perimeter in $(-1,1) \times \mathbb{R}^{2}$. Nevertheless, $b \notin$ $L_{\text {loc }}^{1}\left((-1,1) ; \mathbb{R}^{2}\right)$.

The next result is the key point of the paper, and concerns the regularity of the barycenter. First, we introduce some notation. Given $i=1, \ldots, n-k$, for all $x^{\prime}$ in $\mathbb{R}^{n-k}$ we write $\hat{x}_{i}:=\left(x_{1}, \ldots, x_{i-1}, x_{i+1}, \ldots, x_{n-k}\right)$. If $\Omega \subset \mathbb{R}^{n-k}$ is an open set, $\Omega_{i}$ denotes its projection on $x_{i}=0$. Moreover, if $f$ is a function defined in $\Omega$, we set $f_{\hat{x}_{i}}:=\left.f\right|_{\Omega \cap R_{\hat{x}_{i}}}$, where $R_{\hat{x}_{i}}$ is the straight line passing through $\left(x_{1}, \ldots, x_{i-1}, 0, x_{i+1}, \ldots, x_{n-k}\right)$ and orthogonal to the hyperplane $x_{i}=0$. In order to simplify the notation, we shall drop the subscript $i$ when it is clear from the context.

Theorem 4.3. Let $E \subset \mathbb{R}^{n}$ and let $\Omega \subset \mathbb{R}^{n-k}$ be an open set such that $E$ has finite perimeter in $\Omega \times \mathbb{R}^{k}$, and $E_{x^{\prime}}$ is equivalent to a $k$-dimensional ball for $\mathcal{L}^{n-k}$-a.e. $x^{\prime} \in \Omega$. Assume that conditions (1.3) and (1.4) hold, and fix $i \in\{1, \ldots, n-k\}$. Then, for $\mathcal{H}^{n-k-1}$. a.e. $\hat{x}_{i} \in \Omega_{i}, b_{\hat{x}_{i}} \in W_{\text {loc }}^{1,1}\left(\Omega \cap R_{\hat{x}_{i}}, \mathbb{R}^{k}\right)$ and for $\mathcal{L}^{1}$-a.e. $x_{i} \in \Omega \cap R_{\hat{x}_{i}}$,

$$
b_{\hat{x}_{i}}^{\prime}\left(x_{i}\right)=\frac{1}{L_{\hat{x}_{i}}^{*}\left(x_{i}\right)} \int_{\partial^{*} E_{x^{\prime}}}\left(y-b_{\hat{x}_{i}}\left(x_{i}\right)\right) \frac{v_{i}^{E}\left(x^{\prime}, y\right)}{\left|v_{y}^{E}\left(x^{\prime}, y\right)\right|} d \mathcal{H}^{k-1}(y) .
$$

In addition, when $k=1, b \in W_{\mathrm{loc}}^{1,1}(\Omega)$ and $\|\nabla b\|_{L^{1}\left(\Omega ; \mathbb{R}^{n-1}\right)} \leq P(E ; \Omega \times \mathbb{R}) / 2$. 
Proof. Since our argument is local, we may assume that $\Omega$ is bounded and $E$ has finite perimeter. Note that $L \in W^{1,1}(\Omega)$, by Propositions 3.5 and 3.6. We divide the proof into several steps and we consider only the case $i=1$, the other cases being analogous.

Step 1. Assume $k=n-1$ and $b$ is locally bounded.

With no loss of generality, we may also assume that $L$ coincides with its Lebesgue representative. By (1.4), $L$ is a strictly positive, absolutely continuous function in $\Omega$. Therefore, since $b$ is locally bounded, for any interval $I$ compactly contained in $\Omega$ the set $E \cap\left(I \times \mathbb{R}^{n-1}\right)$ is essentially bounded.

Let us introduce the auxiliary function $f\left(x^{\prime}\right):=\int_{E_{x^{\prime}}} y d y \in \mathbb{R}^{n-1}$. Let $\phi \in C_{c}^{1}(I)$, and let $\left\{\psi_{j}\right\}_{j \in \mathbb{N}}$ be a sequence in $C_{c}^{1}\left(\mathbb{R}^{n-1},[0,1]\right)$ pointwise converging to 1 . Using (2.1) and (1.3), by the dominated convergence theorem and the coarea formula we obtain

$$
\begin{aligned}
-\int_{I} \phi^{\prime}\left(x^{\prime}\right) f & \left(x^{\prime}\right) d x^{\prime}=-\lim _{j \rightarrow \infty} \int_{I \times \mathbb{R}^{n-1}} y \phi^{\prime}\left(x^{\prime}\right) \psi_{j}(y) \chi_{E}\left(x^{\prime}, y\right) d x^{\prime} d y \\
& =\lim _{j \rightarrow \infty} \int_{I \times \mathbb{R}^{n-1}} y \phi\left(x^{\prime}\right) \psi_{j}(y) d D_{1} \chi_{E}(x)=\int_{I \times \mathbb{R}^{n-1}} y \phi\left(x^{\prime}\right) d D_{1} \chi_{E}(x) \\
& =\int_{\partial^{*} E \cap\left(I \times \mathbb{R}^{n-1}\right)} y \phi\left(x^{\prime}\right) v_{1}^{E}\left(x^{\prime}, y\right) d \mathcal{H}^{n-1}(x) \\
& =\int_{I} \phi\left(x^{\prime}\right) d x^{\prime} \int_{\partial^{*} E_{x^{\prime}}} y \frac{v_{1}^{E}\left(x^{\prime}, y\right)}{\left|v_{y}^{E}\left(x^{\prime}, y\right)\right|} d \mathcal{H}^{n-2}(y) .
\end{aligned}
$$

Since $I$ and $\phi$ are arbitrary, $f$ belongs to $W_{\text {loc }}^{1,1}\left(\Omega ; \mathbb{R}^{n-1}\right)$.

Consequently, $b=f / L$ belongs to $W_{\text {loc }}^{1,1}\left(\Omega ; \mathbb{R}^{n-1}\right)$, and by (3.2) and (4.2) we get

$$
\begin{aligned}
b^{\prime}\left(x^{\prime}\right) & =\left(\frac{1}{L} f\right)^{\prime}\left(x^{\prime}\right)=-\frac{L^{\prime}\left(x^{\prime}\right)}{\left|L\left(x^{\prime}\right)\right|^{2}} f\left(x^{\prime}\right)+\frac{1}{L\left(x^{\prime}\right)} \int_{\partial^{*} E_{x^{\prime}}} y \frac{v_{1}^{E}\left(x^{\prime}, y\right)}{\left|v_{y}^{E}\left(x^{\prime}, y\right)\right|} d \mathcal{H}^{n-2}(y) \\
& =-\frac{b\left(x^{\prime}\right)}{L\left(x^{\prime}\right)} \int_{\partial^{*} E_{x^{\prime}}} \frac{v_{1}^{E}\left(x^{\prime}, y\right)}{\left|v_{y}^{E}\left(x^{\prime}, y\right)\right|} d \mathcal{H}^{n-2}(y)+\frac{1}{L\left(x^{\prime}\right)} \int_{\partial^{*} E_{x^{\prime}}} y \frac{v_{1}^{E}\left(x^{\prime}, y\right)}{\left|v_{y}^{E}\left(x^{\prime}, y\right)\right|} d \mathcal{H}^{n-2}(y) \\
& =\frac{1}{L\left(x^{\prime}\right)} \int_{\partial^{*} E_{x^{\prime}}}\left(y-b\left(x^{\prime}\right)\right) \frac{v_{1}^{E}\left(x^{\prime}, y\right)}{\left|v_{y}^{E}\left(x^{\prime}, y\right)\right|} d \mathcal{H}^{n-2}(y) .
\end{aligned}
$$

Step 2. In order to remove the boundedness condition on $b$, we first examine the case $n=2, k=1$.

Our strategy is to shrink the set $E$ in a suitable way: given $M>0$, we translate every segment $E_{x^{\prime}}$ contained in the half-plane $y>M$ (resp. in $y<-M$ ) until it touches the line $y=M$ (resp. $y=-M$ ). To be more precise, defining the truncation $b^{M}:=$ $(b \wedge(L / 2+M)) \vee(-L / 2-M)$, we set

$$
E^{M}:=\bigcup_{x^{\prime} \in \Omega}\left(b^{M}\left(x^{\prime}\right)-L\left(x^{\prime}\right) / 2, b^{M}\left(x^{\prime}\right)+L\left(x^{\prime}\right) / 2\right) .
$$

We want to show that $E^{M}$ has finite perimeter and satisfies condition (1.3). This, thanks to Step 1 , will imply that $b^{M} \in W_{\mathrm{loc}}^{1,1}(\Omega)$. 


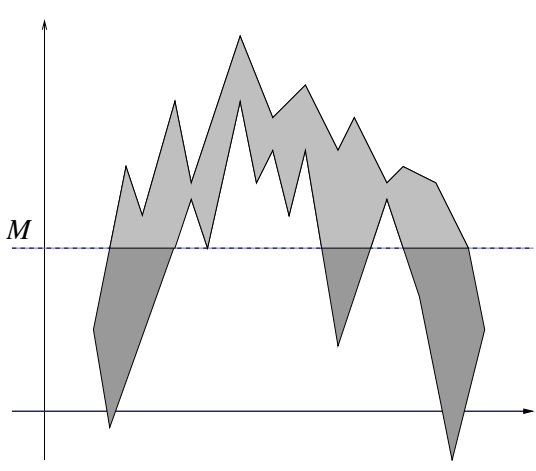

Fig. 4. The sets $E$ and $F_{+}^{M}$.

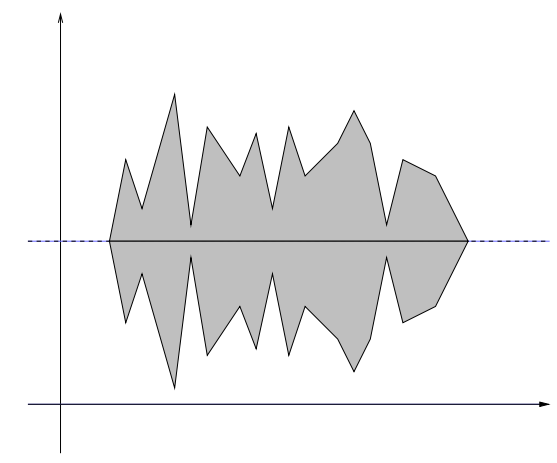

Fig. 6. The set $G$.

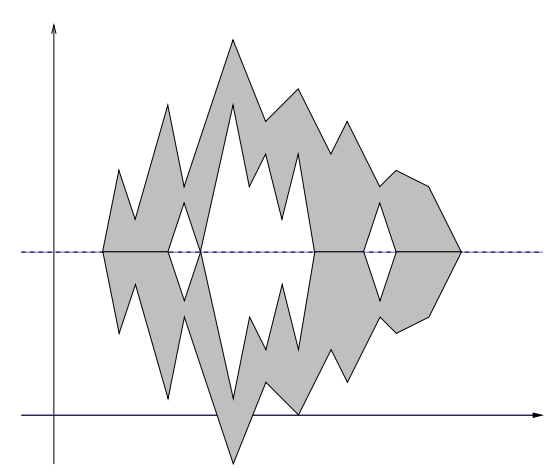

Fig. 5. The set $F_{+}^{M} \cup \widetilde{F}_{+}^{M}$.

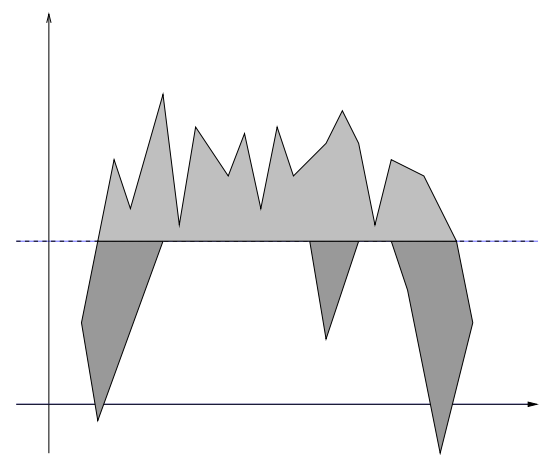

Fig. 7. The sets $G_{+}^{M}$ and $E^{M}$.

We set $F_{+}^{M}:=\left\{\left(x^{\prime}, y\right) \in E: y>M\right\}$ (Figure 4) and consider its reflection with respect to the line $y=M$, i.e., $\widetilde{F}_{+}^{M}:=\left\{\left(x^{\prime}, y\right) \in \mathbb{R}^{2}:\left(x^{\prime}, 2 M-y\right) \in F_{+}^{M}\right\}$ (Figure 5). Let $G$ be the set obtained from $F_{+}^{M} \cup \widetilde{F}_{+}^{M}$ through Steiner symmetrization with respect to $y=M$ (Figure 6), and let $G_{+}^{M}:=\left\{\left(x^{\prime}, y\right) \in G: y>M\right\}$ (Figure 7).

Observing that $\left(F_{+}^{M} \cup \widetilde{F}_{+}^{M}\right)^{(1 / 2)} \cap\{y=M\} \subset E^{(1 / 4)}$ and that by Theorem 2.1, $\mathcal{H}^{1}\left(E^{(1 / 4)}\right)=0$, we have $P\left(F_{+}^{M} \cup \widetilde{F}_{+}^{M}\right) \leq 2 P(E)$. Bearing in mind that Steiner symmetrization decreases the perimeter, we get $P(G) \leq 2 P(E)$. Since all sections $G_{x^{\prime}}$ of $G$ are segments, it is easy to verify that $\partial^{*} G_{+}^{M} \cap\{y=M\}$ is contained (up to an $\mathcal{H}^{1}$-negligible set) in the projection of $\partial^{*} G \cap\{y>M\}$ on $\{y=M\}$. Therefore, $P\left(G_{+}^{M}\right) \leq P(G) \leq 2 P(E)$.

Let us now repeat the same procedure on the set $F_{-}^{M}:=\left\{\left(x^{\prime}, y\right) \in E: y<-M\right\}$ in a symmetric way with respect to the line $y=-M$, thus obtaining another set $G_{-}^{M}$ such that $P\left(G_{-}^{M}\right) \leq 2 P(E)$. Since by construction

$$
E^{M}=\left(E \backslash\left(F_{+}^{M} \cup F_{-}^{M}\right)\right) \cup G_{+}^{M} \cup G_{-}^{M},
$$

we infer that $P\left(E^{M}\right) \leq 5 P(E)$ and condition (1.3) is still satisfied thanks to Proposition 3.6. 
Define now the functions

$$
h_{ \pm}\left(x^{\prime}\right):=b\left(x^{\prime}\right) \pm L\left(x^{\prime}\right) / 2 \quad \text { and } \quad h_{ \pm}^{M}\left(x^{\prime}\right):=b^{M}\left(x^{\prime}\right) \pm L\left(x^{\prime}\right) / 2 .
$$

From the Poincaré-Wirtinger inequality in $W^{1,1}(I)$,

$$
\int_{I}\left|h_{ \pm}^{M}-f_{I} h_{ \pm}^{M}\right| d x^{\prime} \leq c(I) \int_{I}\left|\left(h_{ \pm}^{M}\right)^{\prime}\right| d x^{\prime},
$$

and the bound $\int_{I}\left|\left(h_{+}^{M}\right)^{\prime}\right|+\left|\left(h_{-}^{M}\right)^{\prime}\right| d x^{\prime} \leq P\left(E^{M}\right) \leq 5 P(E)$, we see that, up to a (not relabeled) subsequence, $h_{ \pm}^{M}-f_{I} h_{ \pm}^{M}$ converges in $\mathrm{BV}(I)$ as $M \rightarrow \infty$. Considering that $h_{ \pm}^{M} \rightarrow h_{ \pm}$pointwise, $h_{ \pm}$belong to $\mathrm{BV}(I)$, and so does $b$. Since $b$ is locally bounded, by Step 1 we conclude that $b \in W_{\text {loc }}^{1,1}(\Omega)$ and (4.1) holds.

Step 3. Now we turn to the case $n>2, k=n-1$. Here our strategy is to rearrange the set $E$, keeping fixed the $(n-1)$-th component of the barycenter, so that the $(n-1)$ dimensional balls $E_{x^{\prime}}$ are all centered in the plane $y_{1}=\cdots=y_{n-2}=0$.

We keep the same definition of $E^{S_{i}}$ used in the proof of Proposition 3.6. A pictorial example of $E^{S_{1}}$ is given in Figure 8. From (3.20) and Proposition 3.6 we deduce that $\mathcal{H}^{n-1}\left(\left\{x \in \partial^{*} E^{S_{1}}: v_{y_{1}}^{E_{1}}=0\right\} \cap\left(\Omega \times \mathbb{R}^{n-1}\right)\right)=0$ and a fortiori (1.3) holds with $E^{S_{1}}$ in place of $E$. Moreover, for $\mathcal{L}^{1}$-a.e. $x^{\prime} \in \Omega$ the section $\left(E^{S_{1}}\right)_{x^{\prime}}$ is a ball obtained by translating $E_{x^{\prime}}$. This fact implies in particular that moreover $E^{S_{1}}$ satisfies (1.4) and that for a.e. $x^{\prime} \in \Omega$ the barycenter of the section $\left(E^{S_{1}}\right)_{x^{\prime}}$ is given by $\left(0, b_{2}\left(x^{\prime}\right), \ldots, b_{n-1}\left(x^{\prime}\right)\right)$.

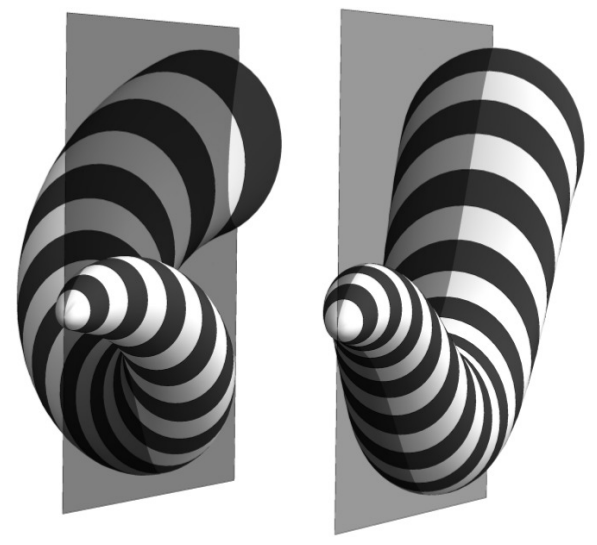

Fig. 8. The sets $E$ (left) and $E^{S_{1}}$ (right)

Iterating this procedure with respect to the $n-3$ variables $y_{2}, \ldots, y_{n-2}$ we obtain the set $E^{S_{n-2}}$, which is equivalent in $\Omega \times \mathbb{R}^{n-1}$ to

$$
F:=\left\{\left(x^{\prime}, y\right) \in \Omega \times \mathbb{R}^{n-1}: x^{\prime} \in \Omega, y \in B_{r\left(x^{\prime}\right)}^{n-1}\left(\left(0, \ldots, 0, b_{n-1}\left(x^{\prime}\right)\right)\right)\right\},
$$

with $\mathcal{H}^{n-1}\left(\left\{x \in \partial^{*} F: v_{y_{n-1}}^{F}(x)=0\right\} \cap\left(\Omega \times \mathbb{R}^{n-1}\right)\right)=0$. From Theorem 2.4, for $\mathcal{L}^{n-2}$ a.e. $y^{\prime}=\left(y_{1}, \ldots, y_{n-2}\right) \in \mathbb{R}^{n-2}$ the section $F_{y^{\prime}}:=\left\{\left(x^{\prime}, y_{n-1}\right) \in \mathbb{R}^{2}:\left(x^{\prime}, y^{\prime}, y_{n-1}\right) \in F\right\}$ 
has finite perimeter. Moreover,

$$
\mathcal{H}^{1}\left(\left\{\left(x^{\prime}, y_{n-1}\right) \in \partial^{*} F_{y^{\prime}}: v_{y_{n-1}}^{F_{y^{\prime}}}\left(x^{\prime}, y_{n-1}\right)=0\right\} \cap(\Omega \times \mathbb{R})\right)=0 .
$$

Indeed, setting $A:=\left\{x \in \partial^{*} F: v_{y_{n-1}}^{F}(x)=0\right\} \cap\left(\Omega \times \mathbb{R}^{n-1}\right)$, by the coarea formula we have $\mathcal{H}^{1}\left(A_{y^{\prime}}\right)=0$ for $\mathcal{L}^{n-2}$-a.e. $y^{\prime}$, while again by Theorem 2.4 the section $A_{y^{\prime}}$ is equivalent to $\left\{\left(x^{\prime}, y_{n-1}\right) \in \partial^{*} F_{y^{\prime}}: v_{y_{n-1}} F_{y^{\prime}}\left(x^{\prime}, y_{n-1}\right)=0\right\} \cap(\Omega \times \mathbb{R})$.

Let $I$ be an interval compactly included in $\Omega$. Since $r=r\left(x^{\prime}\right)$ is continuous, there exists a $\rho>0$ such that $I \times B_{\rho}^{n-2}$ is included in the projection of $F$ on the hyperplane $y_{n-1}=0$. For all $y^{\prime} \in B_{\rho}^{n-2}$ and $x^{\prime} \in I$ the 1-dimensional section $\left(F_{y^{\prime}}\right)_{x^{\prime}}$ of $F_{y^{\prime}}$ is a segment of $\mathcal{L}^{1}$-measure equal to $2 \sqrt{r\left(x^{\prime}\right)^{2}-\left|y^{\prime}\right|^{2}}>0$ and its barycenter coincides with $b_{n-1}\left(x^{\prime}\right)$. Therefore, $b_{n-1} \in W_{\text {loc }}^{1,1}(\Omega)$ by Step 2 and (4.1) holds by Step 1 .

Step 4. The remaining case, i.e., $k<n-1$, will be addressed through a slicing argument.

Let $Q=\prod_{i=1}^{n-k} I_{i}$ be a cube compactly included in $\Omega$. For any $\hat{x} \in \prod_{i=2}^{n-k} I_{i}$ set $E_{\hat{x}}=$ $\left\{\left(x_{1}, y\right) \in \mathbb{R}^{k+1}:\left(x_{1}, \hat{x}, y\right) \in E\right\}$. Let us now prove that for $\mathcal{L}^{n-k-1}$-a.e. $\hat{x} \in \prod_{i=2}^{n-k} I_{i}$ the slice $E_{\hat{x}} \subset \mathbb{R}^{k+1}$ satisfies the same assumptions of the sets considered in Steps 2 and 3. Indeed, the section $E_{\hat{x}}$ has finite perimeter in $\mathbb{R}^{k+1}$ thanks to Theorem 2.4, and $\left(E_{\hat{x}}\right)_{x_{1}}=$ $E_{\left(x_{1}, \hat{x}\right)}$ is a $k$-dimensional ball for $\mathcal{L}^{1}$-a.e. $x_{1} \in I_{1}$. Moreover, the same argument used to prove (4.3) shows that $\mathcal{H}^{k}\left(\left\{\left(x_{1}, y\right) \in \partial^{*} E_{\hat{x}}: v_{y}^{E_{\hat{x}}}\left(x_{1}, y\right)=0\right\} \cap\left(I_{1} \times \mathbb{R}^{k}\right)\right)=0$. Finally, by [1, Theorem 3.108], $\left(L^{*}\right)_{\hat{x}}$ is the Lebesgue representative of $L_{\hat{x}}$. Therefore, $\left(L^{*}\right)_{\hat{x}}>0$ in $I_{1}$, given that the projection on $\prod_{i=2}^{n-k} I_{i}$ of the set $\left\{x^{\prime} \in \Omega: L^{*}\left(x^{\prime}\right)=0\right\}$ is $\mathcal{L}^{n-k-1}$-negligible.

By Steps 2 and 3 we conclude that $b_{\hat{x}} \in W^{1,1}\left(I_{1} ; \mathbb{R}^{k}\right)$. Using again Theorem 2.4 we get (4.1):

$$
\begin{aligned}
b_{\hat{x}}^{\prime}\left(x_{1}\right) & =\frac{1}{L_{\hat{x}}^{*}\left(x_{1}\right)} \int_{\partial^{*}\left(E_{\hat{x}}\right)_{x_{1}}}\left(y-b_{\hat{x}}\left(x_{1}\right)\right) \frac{v_{1}^{E_{\hat{x}}}\left(x_{1}, y\right)}{\left|v_{y}^{E_{\hat{x}}}\left(x_{1}, y\right)\right|} d \mathcal{H}^{k-1}(y) \\
& =\frac{1}{L^{*}\left(x_{1}, \hat{x}\right)} \int_{\left(\partial^{*} E\right)_{\left(x_{1}, \hat{x}\right)}}\left(y-b\left(x_{1}, \hat{x}\right)\right) \frac{v_{1}^{E}\left(x_{1}, \hat{x}, y\right)}{\left|v_{y}^{E}\left(x_{1}, \hat{x}, y\right)\right|} d \mathcal{H}^{k-1}(y) .
\end{aligned}
$$

Step 5. We shall now consider the special case $k=1$, to prove that $b \in W_{\mathrm{loc}}^{1,1}(\Omega)$ and $\|\nabla b\|_{L^{1}\left(\Omega ; \mathbb{R}^{n-1}\right)} \leq P(E ; \Omega \times \mathbb{R}) / 2$. From (4.1), we get

$$
\begin{aligned}
\int_{\prod_{i=2}^{n-1} I_{i}} d \hat{x} \int_{I_{1}}\left|b_{\hat{x}}^{\prime}\left(x_{1}\right)\right| d x_{1} & \leq \frac{1}{2} \int_{Q} d x^{\prime} \int_{\left(\partial^{*} E\right)_{x^{\prime}}} \frac{\left|v_{1}^{E}\left(x^{\prime}, y\right)\right|}{\left|v_{y}^{E}\left(x^{\prime}, y\right)\right|} d \mathcal{H}^{0}(y) \\
& \leq \frac{1}{2} P(E ; Q \times \mathbb{R}) .
\end{aligned}
$$

Clearly, the same bound holds if we consider the slices of $b$ with respect to all the remaining directions. Fix now $T>0$ and consider the truncated function $b^{T}:=(b \wedge T) \vee(-T)$. From (4.4) we have

$$
\int_{Q}\left|\left(b_{\hat{x}}^{T}\right)^{\prime}\right| d x_{1} d \hat{x} \leq \frac{1}{2} P(E ; Q \times \mathbb{R}) .
$$


The same holds for the other directions. As a consequence of [1, Theorem 3.105], $b^{T}$ belongs to $W^{1,1}(Q)$. Therefore, by using the Poincaré-Wirtinger inequality as in Step 2 and letting $T \rightarrow \infty$, we get $b \in W^{1,1}(Q)$. Finally, from [1, Theorem 3.107] it follows that for $\mathcal{L}^{n-1}$-a.e. $x^{\prime} \in \Omega$,

$$
\nabla b\left(x^{\prime}\right)=\frac{1}{L\left(x^{\prime}\right)} \int_{\partial^{*} E_{x^{\prime}}}\left(y-b\left(x^{\prime}\right)\right) \frac{v_{x^{\prime}}^{E}\left(x^{\prime}, y\right)}{\left|v_{y}^{E}\left(x^{\prime}, y\right)\right|} d \mathcal{H}^{0}(y) .
$$

In particular, this implies $\|\nabla b\|_{L^{1}\left(\Omega ; \mathbb{R}^{n-1}\right)} \leq P(E ; \Omega \times \mathbb{R}) / 2$.

The next example shows that in general the regularity of the barycenter is only local.

Example 4.4. Let $n=3, k=2$. Let $\Omega=\pi^{+}(E)=(0,1)$, and let

$$
E=\left\{\left(x^{\prime}, y\right) \in \mathbb{R} \times \mathbb{R}^{2}:\left|y-b\left(x^{\prime}\right)\right|<r\left(x^{\prime}\right), x^{\prime} \in(0,1)\right\},
$$

where $r\left(x^{\prime}\right)=\left|x^{\prime}\right|^{2}$ and $b:(0,1) \rightarrow \mathbb{R}^{2}$ is given by

$$
b\left(x^{\prime}\right):=\left(0, \sin \left(1 / x^{\prime}\right)\right), \quad x^{\prime} \in(0,1) .
$$

We observe that $L\left(x^{\prime}\right)=\pi\left|x^{\prime}\right|^{4}$ is strictly positive in $(0,1)$. Moreover,

$$
\begin{aligned}
P\left(E ;(0,1) \times \mathbb{R}^{2}\right) & =\int_{0}^{1} r\left(x^{\prime}\right) d x^{\prime} \int_{0}^{2 \pi} \sqrt{1+\left|b^{\prime}\left(x^{\prime}\right) \cdot(\cos \theta, \sin \theta)+r^{\prime}\left(x^{\prime}\right)\right|^{2}} d \theta \\
& \leq 2 \pi \int_{0}^{1} r\left(x^{\prime}\right) \sqrt{1+2\left|b^{\prime}\left(x^{\prime}\right)\right|^{2}+2\left|r^{\prime}\left(x^{\prime}\right)\right|^{2}} d x_{1}<\infty,
\end{aligned}
$$

so that $E$ is of finite perimeter in $(0,1) \times \mathbb{R}^{2}$. In addition, $L \in W^{1,1}(0,1)$ and conditions (1.3) and (1.4) are satisfied. Nevertheless, $b \notin W^{1,1}\left((0,1) ; \mathbb{R}^{2}\right)$.

The next example shows that in codimension higher than 1, despite being absolutely continuous on almost every 1 -dimensional section of $\Omega$, the barycenter may not be in $W_{\text {loc }}^{1,1}\left(\Omega ; \mathbb{R}^{2}\right)$.

Example 4.5. Let $n=4, k=2$. Let $L: B_{1}^{2} \rightarrow[0, \infty)$ be a continuous function vanishing only at the origin, and such that $L \in W^{1,1}\left(B_{1}^{2}\right)$, while $\sqrt{L}$ does not belong to $\mathrm{BV}(U)$ for any neighborhood $U$ of the origin. For example, one can take $L\left(x^{\prime}\right)=$ $\left|x^{\prime}\right|^{5 / 4}\left[2+\sin \left(1 /\left|x^{\prime}\right|^{2}\right)\right]$. Set

$$
E=\left\{\left(x^{\prime}, y\right) \in \mathbb{R}^{2} \times \mathbb{R}^{2}:\left|y-b\left(x^{\prime}\right)\right|<r\left(x^{\prime}\right), x^{\prime} \in B_{1}^{2}\right\},
$$

where $r\left(x^{\prime}\right)=\sqrt{L\left(x^{\prime}\right) / \pi}$ and $b\left(x^{\prime}\right):=\left(0, r\left(x^{\prime}\right)\right)$. Then

$$
P\left(E ; B_{1}^{2} \times \mathbb{R}^{2}\right)=\int_{B_{1}^{2}} r\left(x^{\prime}\right) d x^{\prime} \int_{0}^{2 \pi} \sqrt{1+\left|\nabla r\left(x^{\prime}\right)\right|^{2}(1+\sin \theta)^{2}} d \theta<\infty,
$$

so that $E$ is of finite perimeter. Note that conditions (1.3) and (1.4) are satisfied. Nevertheless, $b \notin \mathrm{BV}_{\text {loc }}\left(B_{1}^{2} ; \mathbb{R}^{2}\right)$.

Once Theorem 4.3 is established, Theorem 1.2 follows at once. 
Proof of Theorem 1.2. By Theorem 1.1, for $\mathcal{L}^{n-k}$-a.e. $x^{\prime} \in \Omega$ the section $E_{x^{\prime}}$ is a $k$ dimensional ball, and $v_{x^{\prime}}^{E}\left(x^{\prime}, \cdot\right)$ and $\left|v_{y}^{E}\right|\left(x^{\prime}, \cdot\right)$ are constant $\mathcal{H}^{k-1}$-a.e. in $\partial^{*} E_{x^{\prime}}$. Moreover, condition (1.3) holds due to Proposition 3.6. Let $i=1, \ldots, n-k$. By (4.1), for $\mathcal{H}^{n-k-1}$ a.e. $\hat{x}_{i} \in \Omega_{i}$ and for $\mathcal{L}^{1}$-a.e. $x_{i} \in \Omega \cap R_{\hat{x}_{i}}$,

$$
b_{\hat{x}_{i}}^{\prime}\left(x_{i}\right)=\frac{1}{L_{\hat{x}_{i}}^{*}\left(x_{i}\right)} \frac{v_{i}^{E}\left(x^{\prime}\right)}{\left|v_{y}^{E}\left(x^{\prime}\right)\right|} \int_{\partial^{*} E_{x^{\prime}}}\left(y-b_{\hat{x}_{i}}\left(x_{i}\right)\right) d \mathcal{H}^{k-1}(y)=0,
$$

where we dropped the variable $y$ for functions that are constant in $\partial^{*} E_{x^{\prime}}$. Arguing as in Step 5 of the proof of Theorem 4.3 , we conclude that $b \in W_{\mathrm{loc}}^{1,1}\left(\Omega ; \mathbb{R}^{k}\right), \nabla b=0$ in $\Omega$, and therefore $b$ is constant in $\Omega$.

\section{Quantitative estimates in the convex case}

The present section focuses on a quantitative version of inequality (1.1) in the case of convex sets, in the spirit of $[8,11,13]$. Firstly, we need a Poincaré type inequality, a particular case of a more general one proved in [9]. We provide the proof for completeness.

Proposition 5.1 (Weighted Poincaré inequality). Let $\Omega \subset \mathbb{R}^{n}, n \geq 1$, be an open set, and let $\bar{x}, \tilde{x} \in \Omega$ and $0<r \leq R<\infty$ be such that $B_{r}(\bar{x}) \subset \Omega \subset B_{R}(\widetilde{x})$. Assume that $\Omega$ is star-shaped with respect to $B_{r}(\bar{x})$. Then

$$
\int_{\Omega}\left|f(x)-f_{B_{r / 2}(\bar{x})}\right| d x \leq\left(\frac{4 R}{r}\right)^{n+1} \int_{\Omega}|\nabla f(x)| \operatorname{dist}(x, \partial \Omega) d x \quad \text { for all } f \in W^{1,1}(\Omega),
$$

where $f_{B_{r / 2}(\bar{x})}$ denotes the average of $f$ on $B_{r / 2}(\bar{x})$.

Proof. Without any loss of generality, we can assume that $\bar{x}=0$. We can also assume that $f \in C^{1}$, since the general case can be obtained by a density argument. For every $y \in \Omega$ and $z \in B_{r / 2}$ we have

$$
f(z)-f(y)=\int_{0}^{1} \nabla f((1-s) y+s z) \cdot(z-y) d s .
$$

Multiplying by $\frac{1}{\mathcal{L}^{n}\left(B_{r / 2}\right)} \chi_{B_{r / 2}}(z)$ and integrating over $z$ gives

$$
f_{B_{r / 2}}-f(y)=\frac{1}{\mathcal{L}^{n}\left(B_{r / 2}\right)} \int_{\Omega} \int_{0}^{1} \nabla f((1-s) y+s z) \cdot(z-y) \chi_{B_{r / 2}}(z) d s d z .
$$

Making the change of variable

$$
x=(1-s) y+s z,
$$

we get

$$
f_{B_{r / 2}}-f(y)=\frac{1}{\mathcal{L}^{n}\left(B_{r / 2}\right)} \int_{\Omega} \int_{0}^{1} \nabla f(x) \cdot \frac{x-y}{s} \chi_{B_{r / 2}}\left(\frac{x-(1-s) y}{s}\right) \frac{1}{s^{n}} d s d x .
$$


Passing to the absolute value and integrating with respect to $y$ yields

$$
\begin{aligned}
\int_{\Omega} \mid f(y) & -f_{B_{r / 2}} \mid d y \\
\leq & \frac{1}{\mathcal{L}^{n}\left(B_{r / 2}\right)} \int_{\Omega} \int_{\Omega} \int_{0}^{1}|\nabla f(x)| \chi_{B_{r / 2}}\left(\frac{x-(1-s) y}{s}\right) \frac{|x-y|}{s^{n+1}} d s d x d y
\end{aligned}
$$

Let us show that

$$
|x-y| \leq \frac{4 R}{r} \operatorname{dist}(x, \partial \Omega) .
$$

This inequality is trivial when $n=1$. Otherwise, if $y \notin B_{r / 2}(z)$, letting $C$ be the convex hull of $B_{r / 2}(z) \cup\{y\}$, we have

$$
|x-y|=\frac{2|z-y|}{r} \operatorname{dist}(x, \partial C) \leq \frac{4 R}{r} \operatorname{dist}(x, \partial \Omega),
$$

because $C \subset \Omega$. Conversely, if $y \in B_{r / 2}(z)$, we have $|x-y| \leq \operatorname{dist}(x, \partial \Omega) \leq$ $\frac{4 R}{r} \operatorname{dist}(x, \partial \Omega)$. Therefore, setting $A(x):=\left\{y \in \Omega:|x-y| \leq \frac{4 R}{r} \operatorname{dist}(x, \partial \Omega)\right\}$ and interchanging the order of integrations yields

$$
\begin{aligned}
& \int_{\Omega}\left|f(y)-f_{B_{r / 2}}\right| d y \\
& \quad \leq \frac{1}{\mathcal{L}^{n}\left(B_{r / 2}\right)} \int_{\Omega}|\nabla f(x)| \int_{A(x)} \int_{0}^{1} \chi_{B_{r / 2}}\left(\frac{x-(1-s) y}{s}\right) \frac{|x-y|}{s^{n+1}} d s d y d x .
\end{aligned}
$$

Also, for $x$ and $y$ fixed, the only values of $s$ which contribute are such that

$$
\frac{|x-y|}{s}-|y| \leq \frac{|x-(1-s) y|}{s} \leq \frac{r}{2}, \quad \text { and so } \quad s \geq \frac{|x-y|}{r / 2+|y|} \geq \frac{|x-y|}{2 R} .
$$

Hence,

$$
\begin{aligned}
\int_{\Omega}\left|f(y)-f_{B_{r / 2}}\right| d y & \leq \frac{1}{\mathcal{L}^{n}\left(B_{r / 2}\right)} \int_{\Omega}|\nabla f(x)| \int_{A(x)}|x-y| \int_{|x-y| /(2 R)}^{1} \frac{1}{s^{n+1}} d s d y d x \\
& =\frac{1}{n \mathcal{L}^{n}\left(B_{r / 2}\right)} \int_{\Omega}|\nabla f(x)| \int_{A(x)} \frac{1}{|x-y|^{n-1}}\left[(2 R)^{n}-|x-y|^{n}\right] d y d x \\
& \leq \frac{(2 R)^{n}}{n \mathcal{L}^{n}\left(B_{r / 2}\right)} \int_{\Omega}|\nabla f(x)| \int_{A(x)} \frac{1}{|x-y|^{n-1}} d y d x \\
& =\frac{(2 R)^{n}}{\mathcal{L}^{n}\left(B_{r / 2}\right)} \int_{\Omega}|\nabla f(x)| \int_{0}^{\frac{4 R}{r} \operatorname{dist}(x, \partial \Omega)} \omega_{n} d \rho d x \\
& =\left(\frac{4 R}{r}\right)^{n+1} \int_{\Omega}|\nabla f(x)| \operatorname{dist}(x, \partial \Omega) d x
\end{aligned}
$$


Corollary 5.2 (see also [6, Theorem 1.1]). Let $\Omega \subset \mathbb{R}^{n}$ be an open, bounded and convex set with eccentricity $\mathcal{E}$, and let $S$ be the ellipsoid with maximum volume included in $\Omega$. Then there is a positive constant $c=c(n)$ such that

$$
\int_{\Omega}\left|f(x)-f_{\widetilde{S}}\right| d x \leq c \mathcal{E} \int_{\Omega}|\nabla f(x)| \operatorname{dist}(x, \partial \Omega) d x \quad \text { for all } f \in W^{1,1}(\Omega),
$$

where $f_{\widetilde{S}}$ denotes the average of $f$ on the ellipsoid $\widetilde{S}:=\bar{x}+(S-\bar{x}) / 2$, with $\bar{x}$ the center of $S$.

Proof. Up to a roto-translation, we can assume that $S=\left\{x \in \mathbb{R}^{n}: \sum_{i=1}^{n}\left(x_{i} / l_{i}\right)^{2}<1\right\}$ for certain $l_{1}, \ldots, l_{n}>0$. Let $\phi: \mathbb{R}^{n} \rightarrow \mathbb{R}^{n}$ be an affine transformation mapping the unit ball $B_{1}$ onto $S$. The matrix associated to $\phi$ is $\left(\delta_{i j} l_{i}\right)$. We set $\widehat{f}=f \circ \phi$ and $\widehat{\Omega}=\phi^{-1}(\Omega)$.

By John's ellipsoid theorem (see [3, Theorem 2.4]) the inclusions $S \subset \Omega \subset n S$ hold. In particular, with $r$ and $R$ denoting the inner and the outer radius of $\Omega$ respectively, we have $\max \left\{l_{i}\right\} \leq R$ and $\min \left\{l_{i}\right\} \geq r / n$. Moreover $B_{1} \subset \widehat{\Omega} \subset B_{n}$. Taking into account that $\operatorname{det} \nabla \phi=\mathcal{L}^{n}(S) / \mathcal{L}^{n}\left(B_{1}\right)$, a change of variables provides

$$
f_{S / 2}:=\frac{1}{\mathcal{L}^{n}(S / 2)} \int_{S / 2} f d x=\frac{1}{\mathcal{L}^{n}(S / 2)} \int_{B_{1 / 2}} \widehat{f} \operatorname{det} \nabla \phi d y=\widehat{f}_{B_{1 / 2}} .
$$

The following estimates hold:

$$
\begin{aligned}
& |\nabla f(\phi(y))|=\left|\nabla \widehat{f}(y)(\nabla \phi(y))^{-1}\right|=\sqrt{\sum_{i} l_{i}^{-2}\left(\partial_{i} \widehat{f}(y)\right)^{2}} \geq \frac{|\nabla \widehat{f}(y)|}{\max \left\{l_{i}\right\}} \geq \frac{|\nabla \widehat{f}(y)|}{R}, \\
& \operatorname{dist}(\phi(y), \partial \Omega)=\inf _{z \in \partial \widehat{\Omega}} \sqrt{\sum_{i} l_{i}^{2}\left(y_{i}-z_{i}\right)^{2}} \geq \min \left\{l_{i}\right\} \operatorname{dist}(y, \partial \widehat{\Omega}) \geq \frac{r}{n} \operatorname{dist}(y, \partial \widehat{\Omega}) .
\end{aligned}
$$

Then, by using Proposition 5.1, we get

$$
\begin{aligned}
\int_{\Omega}\left|f(x)-f_{S / 2}\right| d x & =\int_{\widehat{\Omega}}\left|\widehat{f}(y)-\widehat{f}_{B_{1 / 2}}\right| \operatorname{det} \nabla \phi d y \\
& \leq(4 n)^{n+1} \int_{\widehat{\Omega}}|\nabla \widehat{f}(y)| \operatorname{dist}(y, \partial \widehat{\Omega}) \operatorname{det} \nabla \phi d y \\
& \leq n(4 n)^{n+1} \mathcal{E} \int_{\widehat{\Omega}}|\nabla f(\phi(y))| \operatorname{dist}(\phi(y), \partial \Omega) \operatorname{det} \nabla \phi d y \\
& =c(n) \mathcal{E} \int_{\Omega}|\nabla f(x)| \operatorname{dist}(x, \partial \Omega) d x .
\end{aligned}
$$

Corollary 5.3. Let $\Omega \subset \mathbb{R}^{n}$ be a bounded and connected Lipschitz domain. Given an open and non-empty set $S \subset \subset \Omega$, there is a positive constant $c_{P}$, depending only on $\Omega$ and $S$, such that

$$
\int_{\Omega}\left|f(x)-f_{S}\right| d x \leq c_{P} \int_{\Omega}|\nabla f(x)| \operatorname{dist}(x, \partial \Omega) d x \quad \text { for all } f \in W^{1,1}(\Omega),
$$

where $f_{S}$ denotes the average of $f$ on $S$. 
Proof. We decompose the domain $\Omega$ into a finite number of domains $\Omega^{i}$, each star-shaped with respect to an open ball $B^{i} \subset \subset \Omega^{i}$ (see [16, Section 1.1.9, Lemma 1]). We also consider a connected open set $U \subset \subset \Omega$, having the cone property and such that $S \subset U$ and $B^{i} \subset U$ for every $i$.

By the Poincaré-Wirtinger inequality in $W^{1,1}(U)$ (see [16, Lemma 1.1.11]),

$$
\begin{aligned}
\left|f_{S}-f_{B^{i}}\right| & =\left|\frac{1}{\mathcal{L}^{n}(S)} \int_{S}\left(f-f_{B^{i}}\right) d x\right| \leq \frac{1}{\mathcal{L}^{n}(S)} \int_{U}\left|f-f_{B^{i}}\right| d x \\
& \leq \frac{c_{i}}{\mathcal{L}^{n}(S)} \int_{U}|\nabla f| d x \leq \frac{c_{i}}{\mathcal{L}^{n}(S) \operatorname{dist}(U, \partial \Omega)} \int_{\Omega}|\nabla f| \operatorname{dist}(x, \partial \Omega) d x
\end{aligned}
$$

for suitable constants $c_{i}=c_{i}\left(B^{i}, U\right)$. Then Proposition 5.1 leads to

$$
\begin{aligned}
\int_{\Omega}\left|f-f_{S}\right| d x & \leq \sum_{i}\left[\int_{\Omega^{i}}\left|f-f_{B^{i}}\right| d x+\mathcal{L}^{n}\left(\Omega^{i}\right)\left|f_{S}-f_{B^{i}}\right|\right] \\
& \leq c_{P} \int_{\Omega}|\nabla f| \operatorname{dist}(x, \partial \Omega) d x .
\end{aligned}
$$

Lemma 5.4. Let $E \subset \mathbb{R}^{n}$ be an open, bounded and convex set with inner radius $r$, outer radius $R$ and eccentricity $\mathcal{E}$. Then

$$
\begin{aligned}
& n^{-2 n} \mathcal{L}^{n}(E) r^{-1} \leq P(E) \leq n^{2 n} \mathcal{L}^{n}(E) r^{-1} \\
& P(E) \geq n^{-2 n} \mathcal{L}^{n}(E)^{\frac{n-2}{n-1}} R^{\frac{1}{n-1}} \\
& P(E) \geq n^{-2 n} \mathcal{L}^{n}(E)^{\frac{n-1}{n}} \mathcal{E}^{\frac{1}{n}}
\end{aligned}
$$

Proof. Let $\mathrm{S}$ be the maximum ellipsoid included in $E$. Up to a roto-translation, we can assume that $S=\left\{x \in \mathbb{R}^{n}: \sum_{i=1}^{n}\left(x_{i} / l_{i}\right)^{2}<1\right\}$ with $l_{1} \leq \cdots \leq l_{n}$. Let also $F:=$ $\prod_{i=1}^{n}\left(-l_{i}, l_{i}\right)$. By John's ellipsoid theorem, the inclusions $(1 / \sqrt{n}) \bar{F} \subset S \subset E \subset n S \subset$ $n F$ hold. In particular, by convexity, $(\sqrt{n})^{1-n} P(F) \leq P(E) \leq n^{n-1} P(F)$. Moreover, $l_{1} \leq r \leq n l_{1}$ and $R \leq n l_{n}$.

Trivially we have $2^{n} \prod_{i=2}^{n} l_{i} \leq P(F) \leq n 2^{n} \prod_{i=2}^{n} l_{i}$ and therefore

$$
\begin{aligned}
\frac{1}{n^{2 n} r} & \leq \frac{1}{n^{n}(\sqrt{n})^{n-1} r} \leq \frac{1}{n^{n}(\sqrt{n})^{n-1} l_{1}} \leq \frac{(\sqrt{n})^{1-n}}{n^{n}} \frac{P(F)}{\mathcal{L}^{n}(F)} \\
& \leq \frac{P(E)}{\mathcal{L}^{n}(E)} \leq \frac{n^{n-1}}{(\sqrt{n})^{-n}} \frac{P(F)}{\mathcal{L}^{n}(F)} \leq \frac{(n \sqrt{n})^{n}}{l_{1}} \leq \frac{n^{n+1}(\sqrt{n})^{n}}{r} \leq \frac{n^{2 n}}{r} .
\end{aligned}
$$

Let $\Omega:=\prod_{i=1}^{n-1}\left(-l_{i}, l_{i}\right)$. By the isoperimetric inequality, we get $P(\Omega) \geq$ $(n-1) \omega_{n-1}^{\frac{1}{n-1}} \mathcal{L}^{n-1}(\Omega)^{\frac{n-2}{n-1}}$. Therefore

$$
\frac{P(E)}{\mathcal{L}^{n}(E)^{\frac{n-2}{n-1}}} \geq \frac{(\sqrt{n})^{1-n}}{n^{\frac{n(n-2)}{n-1}}} \frac{P(F)}{\mathcal{L}^{n}(F)^{\frac{n-2}{n-1}}} \geq \frac{2 l_{n} P(\Omega)}{n^{2 n-1}\left(2 l_{n} \mathcal{L}^{n-1}(\Omega)\right)^{\frac{n-2}{n-1}}} \geq \frac{l_{n}^{\frac{1}{n-1}}}{n^{2 n-1}} \geq \frac{R^{\frac{1}{n-1}}}{n^{2 n}} .
$$

Finally, by writing $(5.3)$ as $P(E)^{n-1} \geq n^{2 n(1-n)} \mathcal{L}^{n}(E)^{n-2} R$, and by using the first inequality in (5.2), we obtain (5.4).

We can now prove Theorem 1.4. 
Proof of Theorem 1.4. For simplicity, we set $\Omega=\pi^{+}\left(\stackrel{\circ}{E}^{S}\right)$, and denote by $r$ and $R$ the inner radius and the outer radius of $E^{S}$ respectively. We shall prove that

$$
\inf _{y \in \mathbb{R}} \mathcal{L}^{n}\left(E^{S} \triangle(E+(0, y))\right) \leq c(n) R \mathcal{E}_{S} \sqrt{P(E)-P\left(E^{S}\right)} \sqrt{P(E)} .
$$

First of all, observe that $b \in W^{1,1}(\Omega)$ by Theorem 4.3. Moreover, by taking into account that $\left|v_{y}^{E^{S}}\right|^{-2}=1+\frac{1}{4}|\nabla L|^{2}$ by (3.3), a simple computation shows that

$$
\sqrt{\left(1+\frac{1}{4}|\nabla L|^{2}+|\nabla b|^{2}\right)^{2}-|\nabla L|^{2}|\nabla b|^{2}} \geq 1+\frac{1}{4}|\nabla L|^{2}+\left(2\left|v_{y}^{E^{S}}\right|^{2}-1\right)|\nabla b|^{2} .
$$

Therefore, the difference $P(E)-P\left(E^{S}\right)$ is greater than or equal to

$$
\begin{aligned}
& \int_{\Omega}\left[\sqrt{1+\left|\nabla b+\frac{1}{2} \nabla L\right|^{2}}+\sqrt{1+\left|\nabla b-\frac{1}{2} \nabla L\right|^{2}}-2 \sqrt{1+\frac{1}{4}|\nabla L|^{2}}\right] d x^{\prime} \\
& =2 \int_{\Omega} \frac{\sqrt{\left(1+\frac{1}{4}|\nabla L|^{2}+|\nabla b|^{2}\right)^{2}-(\nabla L \cdot \nabla b)^{2}}-\left(1+\frac{1}{4}|\nabla L|^{2}-|\nabla b|^{2}\right)}{\sqrt{1+\left|\nabla b+\frac{1}{2} \nabla L\right|^{2}}+\sqrt{1+\left|\nabla b-\frac{1}{2} \nabla L\right|^{2}}+2 \sqrt{1+\frac{1}{4}|\nabla L|^{2}}} d x^{\prime} \\
& \geq 4 \int_{\Omega} \frac{\left|v_{y}^{E^{S}}\right|^{2}|\nabla b|^{2}}{D} d x^{\prime}
\end{aligned}
$$

where $D$ denotes the denominator of the second integrand. Also, observe that, by the convexity of $E^{S}$,

$$
\left|v_{y}^{E^{S}}\left(x^{\prime}\right)\right| \geq \frac{\operatorname{dist}\left(x^{\prime}, \partial \Omega\right)}{\sqrt{2} R} \text { for } x^{\prime} \in \Omega .
$$

Indeed, since $\left|v_{y}^{E^{S}}\left(x^{\prime}\right)\right| /\left|v_{x^{\prime}}^{E^{S}}\left(x^{\prime}\right)\right| \geq \operatorname{dist}\left(x^{\prime}, \partial \Omega\right) / R$, if $\left|v_{x^{\prime}}^{E^{S}}\left(x^{\prime}\right)\right| \geq 1 / \sqrt{2}$ then (5.7) follows. On the other hand, if $\left|v_{y}^{E^{S}}\left(x^{\prime}\right)\right| \geq 1 / \sqrt{2}$, then (5.7) is trivial. Using the Hölder inequality we get

$$
\begin{aligned}
& \sqrt{P(E)-P\left(E^{S}\right)} \sqrt{P(E)+P\left(E^{S}\right)}=\sqrt{P(E)-P\left(E^{S}\right)}\left(\int_{\Omega} D d x^{\prime}\right)^{1 / 2} \\
& \geq 2 \int_{\Omega}\left|v_{y}^{E^{S}}\left(x^{\prime}\right)\right|\left|\nabla b\left(x^{\prime}\right)\right| d x^{\prime} \geq \frac{\sqrt{2}}{R} \int_{\Omega}\left|\nabla b\left(x^{\prime}\right)\right| \operatorname{dist}\left(x^{\prime}, \partial \Omega\right) d x^{\prime} .
\end{aligned}
$$

Let $S$ denote the maximum ellipsoid in $\Omega$, and let $b_{0}$ denote the average of $b$ in $\widetilde{S}:=$ $\bar{x}+(S-\bar{x}) / 2$, with $\bar{x}$ the center of $S$. Since the eccentricity of $\Omega$ is smaller than that of $E^{S}$, by using Corollary 5.2, we have

$$
\begin{aligned}
\sqrt{P(E)-P\left(E^{S}\right)} \sqrt{P(E)+P\left(E^{S}\right)} \geq \frac{c(n)}{R \mathcal{E}_{S}} \int_{\Omega}\left|b-b_{0}\right| d x^{\prime} \\
\geq \frac{c(n)}{R \mathcal{E}_{S}} \int_{\Omega} \mathcal{L}^{1}\left(E_{x^{\prime}}^{S} \Delta\left(E_{x^{\prime}}-b_{0}\right)\right) d x^{\prime}=\frac{c(n)}{R \mathcal{E}_{S}} \mathcal{L}^{n}\left(E^{S} \triangle\left(E-\left(0, b_{0}\right)\right)\right),
\end{aligned}
$$

thus obtaining (5.5). 
To conclude the proof, observe that if $D(E)>1$ then $\lambda(E)<2 \sqrt{D(E)}$, since we always have $\lambda(E)<2$. On the other hand, if $D(E) \leq 1$, since $P(E) \leq 2 P\left(E^{S}\right)$, from (5.2) and (5.5) we obtain

$$
\lambda(E) \leq c(n) R \mathcal{E}_{S} \sqrt{D(E)} \frac{P\left(E^{S}\right)}{\mathcal{L}^{n}\left(E^{S}\right)} \leq c(n) \mathcal{E}_{S}^{2} \sqrt{D(E)} .
$$

Example 5.5. Let $E_{m}$ be a right-angle triangle with basis $1 / m$ and height $2 m$ as in Figure 9. It can be computed that $\lambda\left(E_{m}\right)=2 / 5$ for every $m \in \mathbb{N}$. On the other hand, $D\left(E_{m}\right) \rightarrow 0$ as $m \rightarrow \infty$. This shows that the dependence on the eccentricity of the constant on the right-hand side of (1.6) cannot be avoided.

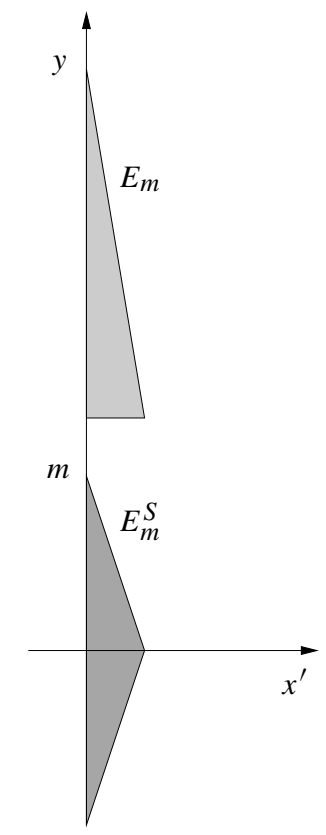

Fig. 9

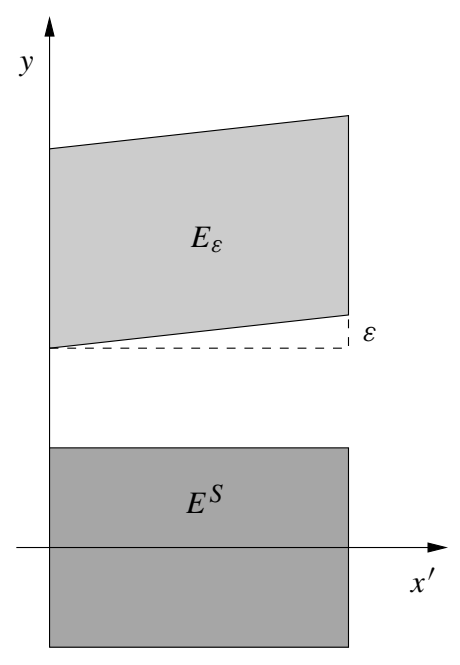

Fig. 10

Example 5.6. Consider the rectangle $E^{S}:=(0, a) \times(-b / 2, b / 2)$ and, for $\varepsilon>0$, the parallelogram $E_{\varepsilon}$ as in Figure 10 . One can compute that $\lambda\left(E_{\varepsilon}\right)=\varepsilon /(2 b)$, and $D\left(E_{\varepsilon}\right) \approx$ $\varepsilon^{2} /\left(2 a^{2}+2 a b\right)$. This shows that the exponent $1 / 2$ in (1.4) is optimal.

Remark 5.7. We can weaken the convexity assumption on $E^{S}$ in Theorem 1.4 by merely requiring that $\left|v_{y}^{E^{S}}\right| \geq c^{\prime} \operatorname{dist}(\cdot, \partial \Omega)$ for some $c^{\prime}=c^{\prime}\left(E^{S}\right)$, and (using Corollary 5.3) that $\Omega=\pi^{+}\left(E^{S}\right)$ is a bounded and connected Lipschitz domain. In this case we get a generic constant $c=c\left(n, E^{S}\right)$ instead of $c(n) \mathcal{E}_{S}^{2}$ on the right-hand side of (1.6). In the example shown in Figure 2 the constant $c$ blows up because $c^{\prime}$ does, as the slope of the dotted segments increases. On the other hand, in the example in Figure 3, the constant $c_{P}$ in (5.1) blows up when the neck in $\Omega=\pi^{+}(E)$ (the area colored black) shrinks. 
Remark 5.8. Note that the first integral in (5.6) is equal to $P(E ; \Omega \times \mathbb{R})-P\left(E^{S} ; \Omega \times \mathbb{R}\right)$, with $\Omega=\pi^{+}\left(\stackrel{\circ}{E}^{S}\right)$. Therefore, (1.6) holds in a slightly stronger form, with $D(E)$ replaced by

$$
\frac{1}{P\left(E^{S}\right)}\left[P(E ; \Omega \times \mathbb{R})-P\left(E^{S} ; \Omega \times \mathbb{R}\right)\right] .
$$

Following the terminology introduced in [13], we say that a set $E \subset \mathbb{R}^{n}$ is $n$-symmetric if it is symmetric with respect to the $n$ coordinate hyperplanes. The next lemma shows that for a convex $n$-symmetric set $E$ the asymmetry index $A(E)$ can be obtained by choosing the ball centered at the origin.

Lemma 5.9. Let $E \subset \mathbb{R}^{n}, n \geq 1$, be an $n$-symmetric bounded convex set with $\mathcal{L}^{n}(E)=$ $\mathcal{L}^{n}\left(B_{r}\right)$ for some $r>0$. Then

$$
\min _{x \in \mathbb{R}^{n}} \mathcal{L}^{n}\left(E \triangle B_{r}(x)\right)=\mathcal{L}^{n}\left(E \triangle B_{r}\right) .
$$

Proof. Given $F, G \subset \mathbb{R}^{n}$, by comparing their sections it is easy to prove $\mathcal{L}^{n}\left(F^{S} \cap G^{S}\right)$ $\geq \mathcal{L}^{n}(F \cap G)$ for any $k$-codimensional Steiner symmetrization.

Note now that $E$ is stable for the 1-dimensional Steiner symmetrizations with respect to $x_{1}=0, \ldots, x_{n}=0$. Hence

$$
\mathcal{L}^{n}\left(E \cap B_{r}(x)\right) \leq \mathcal{L}^{n}\left(E \cap B_{r}\left(x_{1}, \ldots, x_{n-1}, 0\right)\right) \leq \cdots \leq \mathcal{L}^{n}\left(E \cap B_{r}\right) .
$$

We can finally prove the quantitative estimate for Steiner symmetrization in the case $2 \leq$ $k \leq n-1$.

Proof of Theorem 1.5.

Step 1. Define $E^{S_{i}}$ as in the proof of Proposition 3.6. Let also $r, R$ and $\mathcal{E}$ be the inner radius, the outer radius and the eccentricity of $E$ respectively. Since Steiner symmetrization decreases the outer radius, while increasing the inner radius, by (5.5) we deduce that for every $i=1, \ldots, k$,

$$
\mathcal{L}^{n}\left(E^{S_{i-1}} \triangle E^{S_{i}}\right) \leq c(n) R \mathcal{E} \sqrt{P\left(E^{S_{i-1}}\right)-P\left(E^{S_{i}}\right)} \sqrt{P\left(E^{S_{i-1}}\right)},
$$

up to a suitable translation in the direction of the $y_{i}$ axis. By the triangle inequality

$$
\begin{aligned}
\mathcal{L}^{n}\left(E \triangle E^{S_{k}}\right) & \leq \sum_{i=1}^{k} \mathcal{L}^{n}\left(E^{S_{i-1}} \triangle E^{S_{i}}\right) \leq c(n) R \mathcal{E} \sqrt{P(E)} \sum_{i=1}^{k} \sqrt{P\left(E^{S_{i-1}}\right)-P\left(E^{S_{i}}\right)} \\
& \leq c(n) R \mathcal{E} \sqrt{P(E)} \sqrt{P(E)-P\left(E^{S}\right)}
\end{aligned}
$$

Step 2. We now estimate the measure of the symmetric difference of $E^{S}$ and $E^{S_{k}}$. To this end, for $\mathcal{L}^{n-k}$-a.e. $x^{\prime} \in \mathbb{R}^{n-k}$ we set

$$
f_{k}\left(x^{\prime}\right):=p_{E^{s_{k}}}^{2}\left(x^{\prime}\right)+\left|\nabla L\left(x^{\prime}\right)\right|^{2}, \quad f\left(x^{\prime}\right):=p_{E^{s}}^{2}\left(x^{\prime}\right)+\left|\nabla L\left(x^{\prime}\right)\right|^{2} .
$$


Using Proposition 3.4, setting $\Omega=\pi^{+}\left(E^{S}\right)$ and using the Hölder inequality, the difference $P\left(E^{S_{k}}\right)-P\left(E^{S}\right)$ can be estimated by

$$
\begin{aligned}
\int_{\Omega}\left(\sqrt{f_{k}\left(x^{\prime}\right)}-\sqrt{f\left(x^{\prime}\right)}\right) d x^{\prime}=\int_{\Omega} \frac{p_{E^{s_{k}}}^{2}\left(x^{\prime}\right)-p_{E^{S}}^{2}\left(x^{\prime}\right)}{\sqrt{f_{k}\left(x^{\prime}\right)}+\sqrt{f\left(x^{\prime}\right)}} d x^{\prime} \\
\quad \geq \frac{\left(\int_{\Omega} \sqrt{p_{E^{S_{k}}}^{2}\left(x^{\prime}\right)-p_{E^{S}}^{2}\left(x^{\prime}\right)} d x^{\prime}\right)^{2}}{\int_{\Omega}\left(\sqrt{f_{k}\left(x^{\prime}\right)}+\sqrt{f\left(x^{\prime}\right)}\right) d x^{\prime}} \geq \frac{\left(\int_{\Omega} \sqrt{p_{E^{S_{k}}}^{2}\left(x^{\prime}\right)-p_{E^{S}}^{2}\left(x^{\prime}\right)} d x^{\prime}\right)^{2}}{2 P\left(E^{S_{k}}\right)} .
\end{aligned}
$$

Thus,

$$
\begin{aligned}
& \sqrt{2 P\left(E^{S_{k}}\right)} \sqrt{P\left(E^{S_{k}}\right)-P\left(E^{S}\right)} \geq \int_{\Omega} \sqrt{p_{E^{S_{k}}}^{2}\left(x^{\prime}\right)-p_{E^{S}}^{2}\left(x^{\prime}\right)} d x^{\prime} \\
& =\int_{\Omega} \sqrt{\frac{p_{E^{S_{k}}}\left(x^{\prime}\right)-p_{E^{S}}\left(x^{\prime}\right)}{p_{E^{S}}\left(x^{\prime}\right)}} \sqrt{p_{E^{S}}\left(x^{\prime}\right)\left(p_{E^{S_{k}}}\left(x^{\prime}\right)+p_{E^{S}}\left(x^{\prime}\right)\right)} d x^{\prime} \\
& \geq \sqrt{2} \int_{\Omega} p_{E^{S}}\left(x^{\prime}\right) \sqrt{\frac{p_{E} S_{k}\left(x^{\prime}\right)-p_{E}\left(x^{\prime}\right)}{p_{E}\left(x^{\prime}\right)}} d x^{\prime} \\
& =\sqrt{2} k \omega_{k} \int_{\Omega} r^{k-1}\left(x^{\prime}\right) \sqrt{\frac{p_{E} s_{k}\left(x^{\prime}\right)-p_{E}\left(x^{\prime}\right)}{p_{E}\left(x^{\prime}\right)}} d x^{\prime} .
\end{aligned}
$$

Thanks to Lemma 5.9, for $\mathcal{L}^{n-k}$-a.e. $x^{\prime} \in \Omega$ we can use the quantitative isoperimetric inequality (1.5) in dimension $k$ to get

$$
\begin{aligned}
\sqrt{P(E)} \sqrt{P(E)-P\left(E^{S}\right)} & \geq \frac{k \omega_{k}}{\gamma_{k}} \int_{\Omega} r^{k-1}\left(x^{\prime}\right) \frac{\mathcal{L}^{n}\left(E_{x^{\prime}}^{S_{k}} \Delta E_{x^{\prime}}^{S}\right)}{\mathcal{L}^{n}\left(E_{x^{\prime}}^{S}\right)} d x^{\prime} \\
& =\frac{k}{\gamma_{k}} \int_{\Omega} \frac{1}{r\left(x^{\prime}\right)} \mathcal{L}^{n}\left(E_{x^{\prime}}^{S_{k}} \Delta E_{x^{\prime}}^{S}\right) d x^{\prime} \geq \frac{k}{\gamma_{k} R} \mathcal{L}^{n}\left(E^{S_{k}} \triangle E^{S}\right) .
\end{aligned}
$$

Combining this estimate with (5.8) and arguing as in the final part of the proof of Theorem 1.4 we obtain

$$
\lambda(E) \leq c(n, k) \mathcal{E}^{2} \sqrt{D(E)} .
$$

Step 3. Let $r_{S}$ and $R_{S}$ be the inner and the outer radii of $E^{S}$ respectively. From (5.2), (5.4) and $\mathcal{L}^{n}\left(E^{S}\right) \leq \omega_{n} R_{S}^{n}$ we obtain

$$
\frac{P(E)}{P\left(E^{S}\right)} \geq \frac{\mathcal{L}^{n}(E)^{(n-1) / n} \mathcal{E}^{1 / n} r_{S}}{n^{4 n} \mathcal{L}^{n}\left(E^{S}\right)} \geq \frac{\mathcal{E}^{1 / n} \mathcal{E}_{S}^{-1}}{n^{4 n} \omega_{n}^{1 / n}} .
$$

Therefore, if $\mathcal{E} \geq 2^{n} n^{4 n^{2}} \omega_{n} \mathcal{E}_{S}^{n}$, we have $P(E) \geq 2 P\left(E^{S}\right)$ and so $\lambda(E) \leq 2 \sqrt{D(E)}$. Otherwise, by (5.9), $\lambda(E) \leq c(n, k) \mathcal{E}_{S}^{2 n} \sqrt{D(E)}$.

Finally, we highlight that for convex sets the measure of the symmetric difference $E \triangle E^{S}$ is equivalent to the Hausdorff distance $d_{\mathcal{H}}\left(E, E^{S}\right)$ between $E$ and $E^{S}$. In the spirit 
of [12], by combining the quantitative isoperimetric inequalities (1.6) and (1.7) with [10, Lemma 4.2], we have the following corollary. Here $\operatorname{diam}(E)$ is the diameter of the set $E$.

Corollary 5.10. Let $1 \leq k \leq n-1$. There exists a constant $c=c(k, n)$ such that, if $E^{S}$ is a Steiner symmetric convex set with eccentricity $\mathcal{E}_{S}$, then, up to a translation in $y$ direction,

$$
d_{\mathcal{H}}\left(E, E^{S}\right) \leq c \operatorname{diam}(E) \mathcal{E}_{S}^{2}(D(E))^{1 /(2 n)}
$$

for every convex set $E \subset \mathbb{R}^{n}$ whose Steiner symmetral is $E^{S}$.

Acknowledgments. This work was supported by the European Research Council 2008 Advanced Grant Analytic Techniques for Geometric and Functional Inequalities, and by the Center for Nonlinear Analysis (NSF grants DMS-0405343 and DMS-0635983). The research of M. Barchiesi was also partially supported by the Spanish Ministry of Science and Innovation (project MTM200907662), and the Basque Department of Education, Universities and Research (project PI2010-01). The research of F. Cagnetti was also partially supported by the Fundação para a Ciência e a Tecnologia through the CMU|Portugal program.

\section{References}

[1] Ambrosio, L., Fusco, N., Pallara, D.: Functions of Bounded Variation and Free Discontinuity Problems. Oxford Math. Monogr., Oxford Univ. Press, New York (2000) Zbl 0957.49001 MR 1857292

[2] Almgren, F. J., Lieb, E. H.: Symmetric decreasing rearrangement is sometimes continuous. J. Amer. Math. Soc. 2, 683-773 (1989) Zbl 0688.46014 MR 1002633

[3] Barvinok, A.: A Course in Convexity. Grad. Stud. Math. 54, Amer. Math. Soc., Providence (2002) Zbl 1014.52001 MR 1940576

[4] Brock, F., Solynin, A. Yu: An approach to symmetrization via polarization. Trans. Amer. Math. Soc. 352, 1759-1796 (2000) Zbl 0965.49001 MR 1695019

[5] Chlebík, M., Cianchi, A., Fusco, N.: The perimeter inequality under Steiner symmetrization: cases of equality. Ann. of Math. 162, 525-555 (2005) Zbl 1087.28003 MR 2178968

[6] Chua, S.-K., Wheeden, R. L.: Weighted Poincaré inequalities on convex domains. Math. Res. Lett. 17, 993-1011 (2010) Zbl 1226.46027 MR 2727624

[7] Cianchi, A., Fusco, N.: Functions of bounded variation and rearrangements. Arch. Ration. Mech. Anal. 165, 1-40 (2002) Zbl 1028.49035 MR 1947097(2003j:49054)

[8] Cicalese, M., Leonardi, G.: A selection principle for the sharp quantitative isoperimetric inequality. Arch. Ration. Mech. Anal. 206, 617-643 (2012) MR 2980529

[9] Drelichman, I., Durán, R. G.: Improved Poincaré inequalities with weights. J. Math. Anal. Appl. 347, 286-293 (2008) Zbl 1154.46016 MR 2433844

[10] Esposito, L., Fusco, N., Trombetti, C.: A quantitative version of the isoperimetric inequality: the anisotropic case. Ann. Scuola Norm. Sup. Pisa Cl. Sci. (5) 4, 619-651 (2005) Zbl 1170.52300 MR 2207737

[11] Figalli, A., Maggi, F., Pratelli, A.: A mass transportation approach to quantitative isoperimetric inequalities. Invent. Math. 182, 167-211 (2010) Zbl 1196.49033 MR 2672283

[12] Fuglede, B.: Stability in the isoperimetric problem for convex or nearly spherical domains in $\mathbb{R}^{n}$. Trans. Amer. Math. Soc. 314, 619-638 (1989) Zbl 0679.52007 MR 0942426

[13] Fusco, N., Maggi, F., Pratelli, A.: The sharp quantitative isoperimetric inequality. Ann. of Math. 168, 941-980 (2008) Zbl 1187.52009 MR 2456887 
[14] Hall, R. R.: A quantitative isoperimetric inequality in $n$-dimensional space. J. Reine Angew. Math. 428, 161-176 (1992) Zbl 0746.52012 MR 1166511

[15] Hall, R. R., Hayman, W. K., Weitsman, A. W.: On asymmetry and capacity. J. Anal. Math. 56, 87-123 (1991) Zbl 0747.31004 MR 1243100

[16] Maz'ja, V. G.: Sobolev Spaces. Springer Ser. Soviet Math., Springer, Berlin (1985) Zbl 0692.46023 MR 0817985

[17] Vol'pert, A. I.: Spaces BV and quasi-linear equations. Math. USSR-Sb. 17, 225-267 (1967) Zbl 0168.07402 MR 0216338 